\title{
Starbursts in isolated galaxies
}

\section{The influence of stellar birth function and IMF}

\author{
Ch. Theis ${ }^{1,4}$ and J. Köppen ${ }^{2,3,4}$ \\ 1 Institute of Astronomy, University of Vienna, Türkenschanzstr. 17, 1180 Vienna, Austria \\ e-mail: theis@astro.univie.ac.at \\ 2 Observatoire Astronomique de Strasbourg, 11 rue de l'Université, 67000 Strasbourg, France \\ e-mail: koppen@astro.u-strasbg.fr \\ 3 International Space University, Parc d'Innovation, 1, rue Jean-Dominique Cassini, 67400 Illkirch-Graffenstaden, France \\ ${ }^{4}$ Institut für Theoretische Physik und Astrophysik, Universität Kiel, 24098 Kiel, Germany
}

Received 26 May 2008 / Accepted 22 April 2009

\section{ABSTRACT}

Context. Starbursts and substantial variations in the star formation histories are a common phenomenon in galaxies. Although predominantly found in interacting galaxies, they also occur in isolated galaxies.

Aims. We study the stability properties of isolated star-forming dwarf galaxies with the aim of identifying starburst modes. The impact of the stellar birth function, i.e. a spontaneous and an induced star formation mode, the initial mass function (IMF), the stellar feedback and the interstellar medium (ISM) model on the galactic star formation history are investigated. We especially focus on dynamically driven starbursts induced by stellar feedback.

Methods. We apply a one-zone model for a star-gas system coupled by both mass and energy transfer. Additionally, we extend the network for active dynamical evolution. This allows for a coupling between the dynamical state of the galaxy and its internal properties, such as star formation activity or the thermal state of the ISM.

Results. While the influence of the dynamics on the total star formation rate is strong, especially with nonlinear stellar birth functions, the coupling of the internal properties (gas temperature) on the dynamics is rather limited, because radiative cooling keeps the gas temperature well below the virial temperature. Because of short cooling and feedback timescales, the star formation rate is close to the equilibrium star formation rates. Quasi-periodic starbursts occur, because star formation follows the variations in the gas density induced by decaying virial oscillations. This behaviour is quite insensitive to the nature and the details of the stellar birth description, viz. whether spontaneous or induced star formation is considered or the IMF is varied. A second type of burst is found as an instability operating when the cooling may drop at very low densities with increasing temperature, in regimes beyond $10^{4} \mathrm{~K}$.

Conclusions. Bursts of star formation occur during transitory phases, when dynamical equilibrium is established. Then they are quasiperiodic on the dynamical timescale. Because of short heating and cooling timescales, the star formation rate follows the equilibrium star formation rate corresponding to the actual gas density.

Key words. galaxies: evolution - galaxies: starburst - ISM: general

\section{Introduction}

Variations in the star formation rate (SFR) in dwarf galaxies have been deduced from several observations like direct measurements of individual star formation histories (Dohm-Palmer et al. 2002), a large variation in the specific SFR, or short gas depletion times (Gallagher \& Hunter 1984). Especially interesting is the group of isolated starburst galaxies, because no external triggers can be invoked for their high activity. Van Zee (2001) studied a large sample of isolated dwarf irregular galaxies. For the majority of these galaxies, she found a fairly constant SFR over the past 10 Gyr indicating that they reached a quasi-equilibrium state burning on a long gas depletion time of about 20 Gyr. However, a small fraction of her sample shows high star formation activity that is strongly concentrated in the central region and/or characterised by small radial scale lengths, i.e. enhanced gas densities. Already Gallagher \& Hunter (1984) stressed that the observation of starburst dIrrs implies a mechanism to organise large-scale star formation which cannot operate all the time due to fast consumption of fuel. On the other hand, the instability leading to an episodic SFR should only work in a small parameter range, because most of the isolated dIrrs seem to evolve in a highly self-regulated manner.

The first theoretical models dealing with star formation variations in galaxies were based on closed-box models. For reasonable models of the interstellar medium (ISM) it turned out that stellar feedback is very effective in suppressing instabilities of the ISM (e.g. Ikeuchi \& Tomita 1983; Scalo \& Struck-Marcell 1986). These models only allow for a burst-like behaviour when a long time-delay (of the order of $10^{8} \mathrm{yrs}$ ) between star formation and stellar feedback is introduced. Recently Quillen \& Bland-Hawthorn (2008) emphasised the importance of such a long time-delay of the feedback for creating episodic star formation in Milky Way type galaxies. The drawback of these models is the long time-delay that exceeds the short feedback timescales related to massive star formation by far. The latter are a few Myr at most (if not quasi-instantaneously when taking stellar winds into account). It is not clear what has kept the ISM from experiencing feedback by stars for such a long time.

Köppen et al. (1995, hereafter KTH95) investigated a simple numerical model motivated by full chemodynamical simulations. They show that the effective SFR is almost independent of 
the detailed recipe for the stellar birth function, provided a negative feedback due to the thermal state of the ISM is considered in the stellar birth function. This also holds for a multi-phase ISM (Köppen et al. 1998). All these box models suffer, however, from neglecting galactic dynamics. Additionally, they are designed for small galactic regions of a few $100 \mathrm{pc}$. As a result, coherence between different unstable regions and global starbursts could not be modelled.

Recently, several attempts have been made to study the evolution of isolated dwarf galaxies by taking full stellar and gas dynamics, as well as star formation and stellar feedback, into account (e.g. Hensler et al. 2004; Pelupessy et al. 2004, 2005). Though these calculations differ in many details, they agree in producing large-scale star formation variations on dynamical timescales in some of their models. This indicates the crucial role dynamics plays for episodic starburst behaviour.

Starbursts are often associated with galaxy interactions. E.g. most of the ultraluminous infrared galaxies are interpreted as the result of galaxy interactions or mergers (e.g. Sanders \& Mirabel 1996). It is interesting that the response of galaxies to interaction-induced perturbations is not unique or simple. Di Matteo et al. (2007) present a subset of merger events created in a numerical survey of galaxy collisions. Their Figs. 8 and 9 show a variety of reaction patterns of the SFR after the first closest approach, ranging from strong starbursts to almost no enhancement in the star formation activity. Another example for dwarf galaxy interactions is presented in Bekki (2008). Despite this model-to-model variation, statistics of large galaxypair samples show a clear correlation between interactions and star formation activity. For example using 12500 galaxy pairs from the SDSS survey Nikolic et al. (2004) find an enhanced star formation rate for projected separations below $50 \mathrm{kpc}$ and an anti-correlation between the projected separation and the star formation rate (for more details, cf. to the review by Struck 2006).

A major disadvantage of the simulations - either for isolated or interacting galaxies - is their complexity. This prevents a detailed investigation of the parameter space and sometimes also hides the key physical mechanisms. Instead of analysing dwarf galaxies by such detailed and complex models, we study here a set of equations that is as simple as possible and that incorporates the main generic features of the complex numerical simulations. The advantage is a (semi-)analytical treatment and a more direct physical interpretation of the implemented physics. Moreover, extended parameter studies can be carried out much more easily with our set of equations than with the full numerical modelling.

This paper introduces an extended version of the analytical models of KTH95 that allows us to deal with the dynamical evolution of a galaxy. By this, the evolution of the star formation activity not only depends on the internal properties of the ISM, but also on the dynamical state of the galaxy. As an application of our analytical model, the star formation activity for dwarf galaxies in the mass regime of a few $10^{9} M_{\odot}$ is studied.

In this paper we investigate the influence on the star formation by studying a generalized stellar birth function (composed of a spontaneous star formation mode with negative thermal feedback and an induced star formation mode), the initial stellar mass function, and the stellar feedback.

This paper is organised as follows. In Sect. 2 we introduce our set of equations. A reference model and the result of parameter variations are presented in Sect. 3. Section 4 contains a discussion of the results.

\section{The numerical model}

\subsection{The non-dynamical closed box model}

Motivated by the complex and computationally expensive chemo-dynamical calculations of Theis et al. (1992) (also used in the simulations for dwarf galaxies by Hensler et al. 2004), KTH95 analysed a set of closed-box models that was designed to be as simple as possible, but still keeps the main qualitative features of the chemo-dynamical network (e.g. feedback processes). These simplified equations describe the temporal evolution of the density of short-lived massive stars $(s)$, of gas $(g)$, and of the internal energy $(e)$ of the gas:

$$
\begin{aligned}
& \frac{\mathrm{d} g}{\mathrm{~d} t}=-\Psi_{\mathrm{b}}(g, T)+\frac{\eta s}{\tau} \\
& \frac{\mathrm{d} s}{\mathrm{~d} t}=\xi \Psi_{\mathrm{b}}(g, T)-\frac{s}{\tau} \\
& \frac{\mathrm{d} e}{\mathrm{~d} t}=h(g) s-g^{2} \Lambda(T) .
\end{aligned}
$$

The gas is consumed by star formation denoted by a stellar birth function $\Psi_{\mathrm{b}}(g, T)$ depending on gas density $g$ and temperature $T^{1}$. The temperature is related to the energy density by $e=b g T$ with $b \equiv 3 / 2 \cdot k / \mu m_{\mathrm{p}}$ ( $k$ is the Boltzmann constant, $\mu$ the mean molecular weight, and $m_{\mathrm{p}}$ the proton mass). The second term in (1) is a source term for the ISM due to stellar winds and supernova ejecta replenishing the ISM. Massive stars are assumed to return a fraction $\eta \approx 90 \%$ of their mass after a mean stellar lifetime $\tau \approx 10$ Myr. Similarly, Eq. (2) describes the formation of massive stars, as well as their death $(\xi \approx 12 \%$ is the mass fraction of massive stars derived from a Salpeter initial mass function (IMF)).

The last equation deals with the energy budget of the ISM gas. Its internal energy is characterised by its temperature $T$. Assuming a turbulence-driven ISM it receives heating mainly by massive stars (e.g. Ly-continuum radiation or type II supernovae). The conversion factor $h$ can be constant, if all the energy is absorbed, or it may depend on gas density, e.g. for radiative heating in an optically thin gas. The main dissipative process of the gas is radiative cooling. The energy loss scales with $g^{2}$, where the proportionality factor $\Lambda(T)$ is the classical cooling function.

The set of equations is closed by a generalized Schmidt-like stellar birth function

$\Psi_{\mathrm{b}}(g, T)=\Psi_{\mathrm{b}, \mathrm{sp}}(g, T) \equiv C_{n} g^{n} \cdot f(T)$.

This function corresponds to spontaneous star formation. (An induced star formation mode will be discussed later in Sect. 3.3.) Different to the classical Schmidt-law we allow for a (negative) thermal feedback

$f(T) \equiv \mathrm{e}^{-T / T_{\mathrm{s}}}$

which reduces the star formation efficiency in case of high gas temperatures ${ }^{2}$. The exponent $n$ is varied between a linear $(n=1)$

\footnotetext{
1 Because the thermal state of all gas components is summarised in a single temperature $T$, this temperature is a "typical" temperature of the gas characterising the thermal energy or pressure of the ISM. This notion is rather similar e.g. to the notion of the external pressure in Blitz \& Rosolowsky (2004).

${ }^{2}$ A negative temperature $T_{\mathrm{s}}$ corresponds to a positive feedback. However, it is related to the gas temperature (velocity dispersion) alone. Therefore, we used a physically better motivated ansatz for positive stellar feedback in Sect. 3.3.
} 
and a quadratic $(n=2)$ Schmidt law; the constant $C_{n}$ is chosen to match the star formation rate observed in the solar neighborhood. Setting $T_{\mathrm{s}}$ to infinity we recover the classical Schmidt law depending only on the gas density.

In case of radiative cooling, thermal equilibrium is quickly established due to short heating and cooling timescales (KTH95). As a result the effective SFR $\Psi_{\mathrm{e}}$ (which allows for a stationary solution of Eqs. (2) and (3)) is independent of the adopted stellar birth function

$\Psi_{\mathrm{e}}\left(g, T_{\mathrm{e}}\right)=g^{2} \frac{\Lambda\left(T_{\mathrm{e}}\right)}{h(g) \xi \tau}$,

where $T_{\mathrm{e}}$ is the equilibrium gas temperature. First, it is remarkable that $\Psi_{\mathrm{e}}$ is between a linear and a quadratic Schmidt-law depending on $h(g)$, but not depending on $\Psi_{\mathrm{b}}$, especially on the exponent $n$. Secondly, star formation depends almost exclusively on gas density. This means that such systems undergo starbursts only when the gas density is strongly enhanced, e.g. by the result of a collapse, an infall, or an induced structural change of the galaxy.

\subsection{Including the dynamics}

We extend our closed-box model for dynamical evolution by considering a volume with characteristic radius $R$ (e.g. the halfmass radius) of the baryonic system of the galaxy. The equation of motion - motivated by the corresponding equation for a thin gaseous shell - is given by

$$
\begin{aligned}
\frac{\mathrm{d}^{2} R}{\mathrm{~d} t^{2}}= & -\left.\frac{\mathrm{d} \Phi_{\mathrm{DM}}}{\mathrm{d} r}\right|_{r=R}-\frac{1}{2} \frac{G M_{\mathrm{b}}}{R^{2}}-\frac{1}{g} \cdot \frac{\mathrm{d} P}{\mathrm{~d} r}+\frac{j^{2}}{R^{3}}-\frac{v_{\text {rad }}}{\tau_{\text {fric }}} \\
= & -\left.\frac{\mathrm{d} \Phi_{\mathrm{DM}}}{\mathrm{d} r}\right|_{r=R}-\frac{1}{2} \frac{G M_{\mathrm{b}}}{R^{2}} \\
& +C_{P} \frac{k}{\mu m_{\mathrm{p}}} \frac{T}{R}+\frac{\left(C_{j} j_{\mathrm{max}}\right)^{2}}{R^{3}}-\frac{v_{\mathrm{rad}}}{C_{\text {fric }} \tau_{\mathrm{ff}}} .
\end{aligned}
$$

The first two terms in (7) describe the gravity, i.e. the contribution of the dark matter potential and the self-gravity of the baryons with the total mass $M_{\mathrm{b}}$. The third term estimates the contribution of the pressure $P$. The fourth term denotes the angular momentum conservation where $j_{\max }$ is the maximum specific angular momentum derived for a circular orbit at the initial radius $R(t=0)=R_{\text {ini. }}$. The last term describes decaying virial oscillations as observed in collapse simulations, and $v_{\text {rad }}$ is the radial velocity $\mathrm{d} R / \mathrm{d} t$. The frictional timescale $\tau_{\text {fric }}=C_{\text {fric }} \tau_{\text {ff }}$ is normalised to the free-fall timescale $\tau_{\text {ff }}$ of the dark matter halo at the initial radius.

From the (half-mass) volume $V(t) \equiv 4 \pi / 3 \cdot R^{3}(t)$ of the system and the mass of each component the densities can be derived, e.g. the gas density reads

$g(t)=\frac{1}{2} \cdot \frac{M_{\mathrm{g}}(t)}{V(t)}$.

Integrating Eqs. (1)-(3) over volume yields the equations for the total masses of the gas, the massive stars, and the total internal energy $E \equiv e V$ inside $R$ :

$$
\begin{aligned}
& \frac{\mathrm{d} M_{\mathrm{g}}}{\mathrm{d} t}=-\Psi(R, g, T)+\frac{\eta M_{\mathrm{s}}}{\tau} \\
& \frac{\mathrm{d} M_{\mathrm{s}}}{\mathrm{d} t}=\xi \Psi(R, g, T)-\frac{M_{\mathrm{s}}}{\tau} \\
& \frac{\mathrm{d} E}{\mathrm{~d} t}=h(g) M_{\mathrm{s}}-\frac{E}{\tau_{\text {cool }}(g, T)}-P \frac{\mathrm{d} V}{\mathrm{~d} t} .
\end{aligned}
$$

The total SFR $\Psi(R, g, T) \equiv \Psi_{\mathrm{b}}(g, T) \cdot V$ (in $M_{\odot} \mathrm{yr}^{-1}$ ) depends not only on the ISM parameters $g$ and $T$, but now also on the current size $R$ of the system. It is worth noting that this applies only for a nonlinear star formation law, because $\Psi(R, g, T)=$ $\Psi_{\mathrm{b}}\left(M_{\mathrm{g}} / V, T\right) \cdot V \propto M_{\mathrm{g}}^{n} \cdot V^{1-n} !$

The temperature is related to the internal energy $E$ by $E=1 / 2 b M_{\mathrm{g}} T$. The factor $1 / 2$ stems from the assumption that $R$ is the half-mass radius. The last term in Eq. (11) denotes the $P \mathrm{~d} V$ work. The pressure $P$ is given by $P=2 / 3 E / V=$ $2 / 3 b g T$. In case of radiative dissipation, the corresponding timescale $\tau_{\text {cool }}(g, T)$ is defined as

$\tau_{\text {cool }}(g, T) \equiv \frac{b T}{g \Lambda(T)}$.

Dissipation by radiative cooling is included by a cooling function combined from Dalgarno \& McCray (1972) for $T<10^{4} \mathrm{~K}$ and Böhringer \& Hensler (1989) for higher temperatures (and a metallicity $\left.Z=1 / 10 Z_{\odot}\right)$.

For the dark matter halo, we apply the universal halo suggested by Burkert (1995) for dwarf galaxies. The scale radius $r_{0}$ of the Burkert halo was set to $8 \mathrm{kpc}$. For a baryonic mass of $M_{\text {gas }}=2 \times 10^{9} M_{\odot}$ and $R_{\text {ini }}=8 \mathrm{kpc}$ the initial baryonic mass fraction is about $10 \%$.

The constants $C_{P}, C_{j}$, and $C_{\text {fric }}$ are adjusted to a $3 \mathrm{~d}-\mathrm{SPH}$ simulation of a collapsing gas sphere in a given static dark matter halo potential. The simulations were done with the SPH part of the code described in Harfst et al. (2006). Two sets of constants were derived: set $1\left(C_{P}=1.91, C_{j}=0.15, C_{\text {fric }}=2.06\right)$ gives a good fit to the early evolution, whereas set $2\left(C_{P}=1.97\right.$, $C_{j}=0.11, C_{\text {fric }}=0.88$ ) describes the late evolution (Fig. 1). The main freedom in the choice of parameters comes from the damping behaviour that is covered by a variation of a factor of 2 in the constant $C_{\text {fric }}$, i.e. in the frictional timescale. When minimising the deviations over all periods the best fit was reached by $C_{P}=1.74, C_{j}=0.13, C_{\text {fric }}=1.64$. If not stated differently, we apply these values.

The Eqs. (7)-(12) yield a closed set of equations including a first approximation for the dynamical evolution. We solve the differential equations using a fourth-order Runge-Kutta integrator with adaptive timestep (Press et al. 1992). The integration accuracy is set to $10^{-10}$ (or better). For convenience we also consider an equation for the mass $M_{\mathrm{r}}$ of the long-lived low-mass stars and the stellar remnants

$\frac{\mathrm{d} M_{\mathrm{r}}}{\mathrm{d} t}=(1-\xi) \Psi(R, g, T)+(1-\eta) \frac{M_{\mathrm{s}}}{\tau}$.

The formation of the low-mass stars is described by the first term and the production of stellar remnants by the second term in Eq. (13). We neglect the gas return by long-lived low-mass stars. This approximation is appropriate for gas-rich systems. Therefore, our models cannot provide a realistic evolution of the gas mass, if the gas fraction is smaller than a few percent. From Eqs. (9) and (10) we get the solution

$M_{\mathrm{r}}(t)=M_{\mathrm{b}}-\left(M_{\mathrm{g}}(t)+M_{\mathrm{s}}(t)\right)$

describing mass conservation, and $M_{\mathrm{b}}$ is the total initial mass in baryons.

A more accurate treatment would include mass return of lowmass stars by planetary nebulae and type Ia supernovae. This would imply the numerically rather expensive computation of the instantaneous mass transfer rates from the details of the past star formation history for the spectrum of stellar masses. For our purpose of studying the general features of galaxy evolution with 

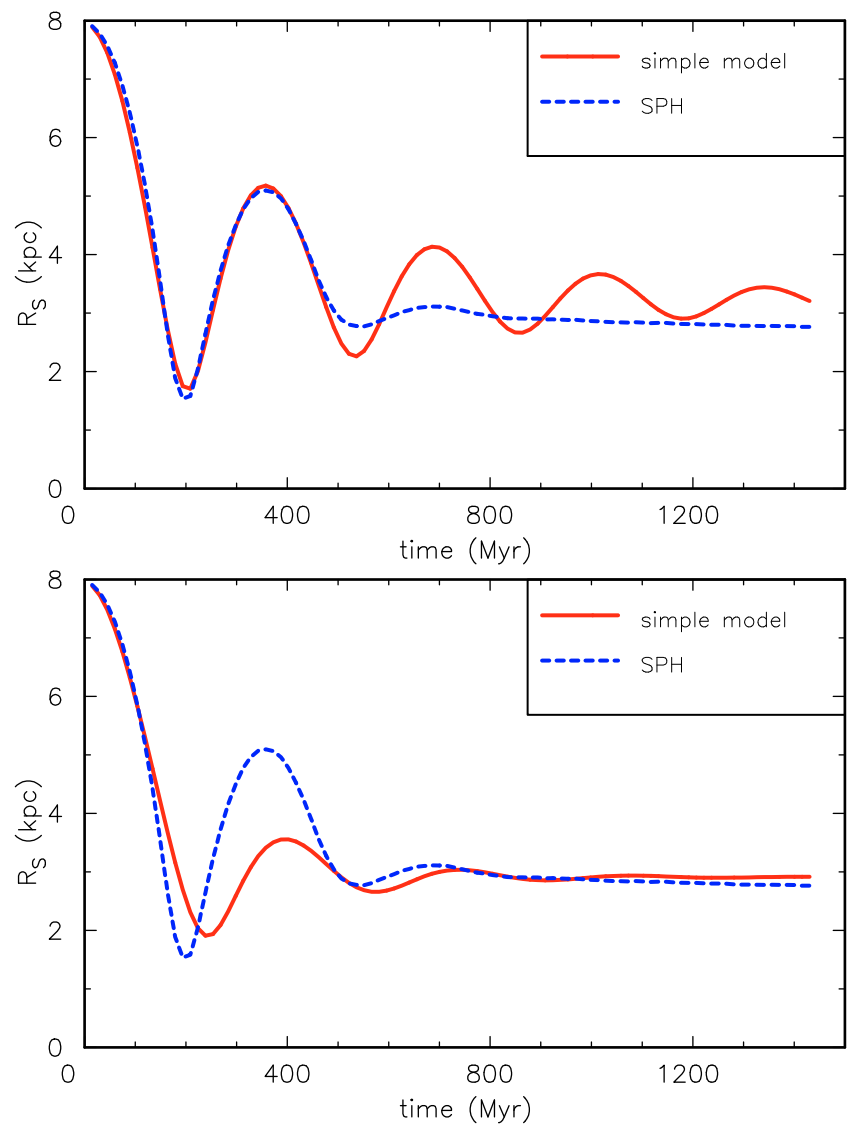

Fig. 1. Temporal evolution of the half-mass radius in a 3D-SPH simulation of a collapsing gas sphere in a dark matter halo and the result for the simple equation of motion (7). Shown are models fitting the early stage well (with $C_{P}=1.91, C_{j}=0.15, C_{f}=2.06$, left) or the late stage (with $C_{P}=1.97, C_{j}=0.11, C_{f}=0.88$, right).

a simplified dynamics, such an elaborate approach would not be such an advantage that it would justify the computational effort.

\subsection{Units}

For our calculations we used the following units: $1 M_{\odot}, 1 \mathrm{pc}$, and 1 Myr. If not stated differently, parameters and results are given in these units or the corresponding derived units, e.g. mass densities in $M_{\odot} \mathrm{pc}^{-3}$.

\section{Results}

\subsection{The reference model}

As a first example we present a model with a total baryonic mass of $2 \times 10^{9} M_{\odot}$ starting at a radius of $R_{\text {ini }}=8 \mathrm{kpc}$. The stellar birth function $\Psi_{\mathrm{b}}$ is parametrised by a Schmidt-exponent of $n=1.5$ and a constant $C_{1.5}=0.06$. Additionally, a feedback term with $T_{\mathrm{s}}=100 \mathrm{~K}$ is considered. The initial temperature is set to $10^{5} \mathrm{~K}$ close to the system's virial temperature

$T_{\mathrm{vir}} \equiv \frac{\mu m_{\mathrm{p}}}{C_{\mathrm{p}} k} \cdot \frac{G\left(M_{\mathrm{DM}}(R)+\frac{1}{2} M_{\mathrm{b}}\right)}{R}$.

This definition is motivated by Eq. (7) assuming force equilibrium between thermal pressure at a temperature $T_{\text {vir }}$ and the
Table 1. Properties of the numerical models.

\begin{tabular}{c|c|c}
\hline \hline Model & SF-law $\left(n, C_{n}, T_{\mathrm{s}}\right)$ & Comment \\
\hline A & $1.5,0.06,10^{2}$ & reference model \\
\hline A2 & $1.5,0.06,10^{4}$ & \\
\hline DN1 & $1.0,0.007, \infty$ & no feedback, Schmidt law \\
DN2 & $1.1,0.007, \infty$ & \\
DN3 & $1.5,0.007, \infty$ & \\
DN4 & $2.0,0.007, \infty$ & \\
DN5 & $2.5,0.007, \infty$ & enhanced $C_{n}$ \\
\hline DN6 & $2.0,0.7, \infty$ & with thermal feedback \\
\hline DT1 & $1.0,0.007,10^{5}$ & \\
DT2 & $1.0,0.007,10^{4}$ & \\
DT3 & $1.0,0.007,10^{3}$ & \\
DT4 & $1.0,0.007,10^{2}$ & quadr. Schmidt law with feedback \\
\hline DT5 & $2.0,0.55,10^{5}$ & induced star formation mode \\
\hline DI1 & ind. SF only & combined SF modes \\
DI2 & model A, $T_{\mathrm{s}}=10^{2}$ & combined SF modes \\
DI3 & model A, $T_{\mathrm{s}}=10^{4}$ & varied constant IMF \\
\hline DX1 & model A, $\xi=6 \%$ & \\
DX2 & model A, $\xi=12 \%$ & Weidner-Kroupa IMF, SN heating \\
\hline DWK1 & $1.5,0.06,10^{2}$ & radiative heating \\
DWK2 & $1.5,0.06,10^{2}$ & \\
\hline
\end{tabular}

gravitational forces of a nonrotating system, and $M_{\mathrm{DM}}(R)$ is the mass of the dark matter component enclosed within the halfmass radius $R$ of the baryonic component. The heating rate is set to $10^{51}$ erg per supernova, i.e. $h=2.4 \times 10^{5} \mathrm{pc}^{2} \mathrm{Myr}^{-3}$.

Figure 2 shows the early evolution: Due to strong cooling, the gas temperature drops almost immediately to values near $10^{4} \mathrm{~K}$ bringing the system far out of virial equilibrium. In the ongoing collapse, the density increases. Cooling and density enhancement continue, until star formation becomes more prominent after about $100 \mathrm{Myr}$. The first stars reinject energy to the ISM leading to the quasi-equilibrium stage known from the box models of KTH95. Different to them, the density increases because of the continuing collapse: the temperature evolves on the line of equilibrium temperatures characteristic of the densities set by the dynamical state of the system. Though the star formation and, by this, the stellar energy injection grow, too, the feedback cannot prevent further collapse, because cooling is still too efficient to allow the gas to reach the virial temperature. The collapse proceeds until $t=0.16 \mathrm{Gyr}$, when the angular momentum conservation in (7) becomes dominant and the system expands again. By this, the first starburst episode comes to an end and the cooling rate drops. The gas is quickly heated to $10^{4} \mathrm{~K}$; however, the gas never reaches the virial temperature thanks to the large increase of the cooling rate at temperatures beyond $10^{4} \mathrm{~K}$. The next star formation cycle starts when the gas is collapsing again.

The longterm evolution of this model shows that the previously discussed bursts are just transitory phenomena caused by the initial departure from dynamical equilibrium (Fig. 3). The oscillations are almost completely damped after 2 Gyr. However, only the first two or three peaks in the SFR are enough strong to be characterised as a starburst by standard definitions. Later on no bursts are found. Such behaviour, i.e. only an initial burst, was found in almost all models.

Two general properties of our models can already be seen in the reference model. First, despite the coupling of stars and gas in the dynamical equation, Eq. (7), the dynamics do (almost) not care about the star formation and the related stellar feedback. This is caused by the very efficient cooling resulting in gas 

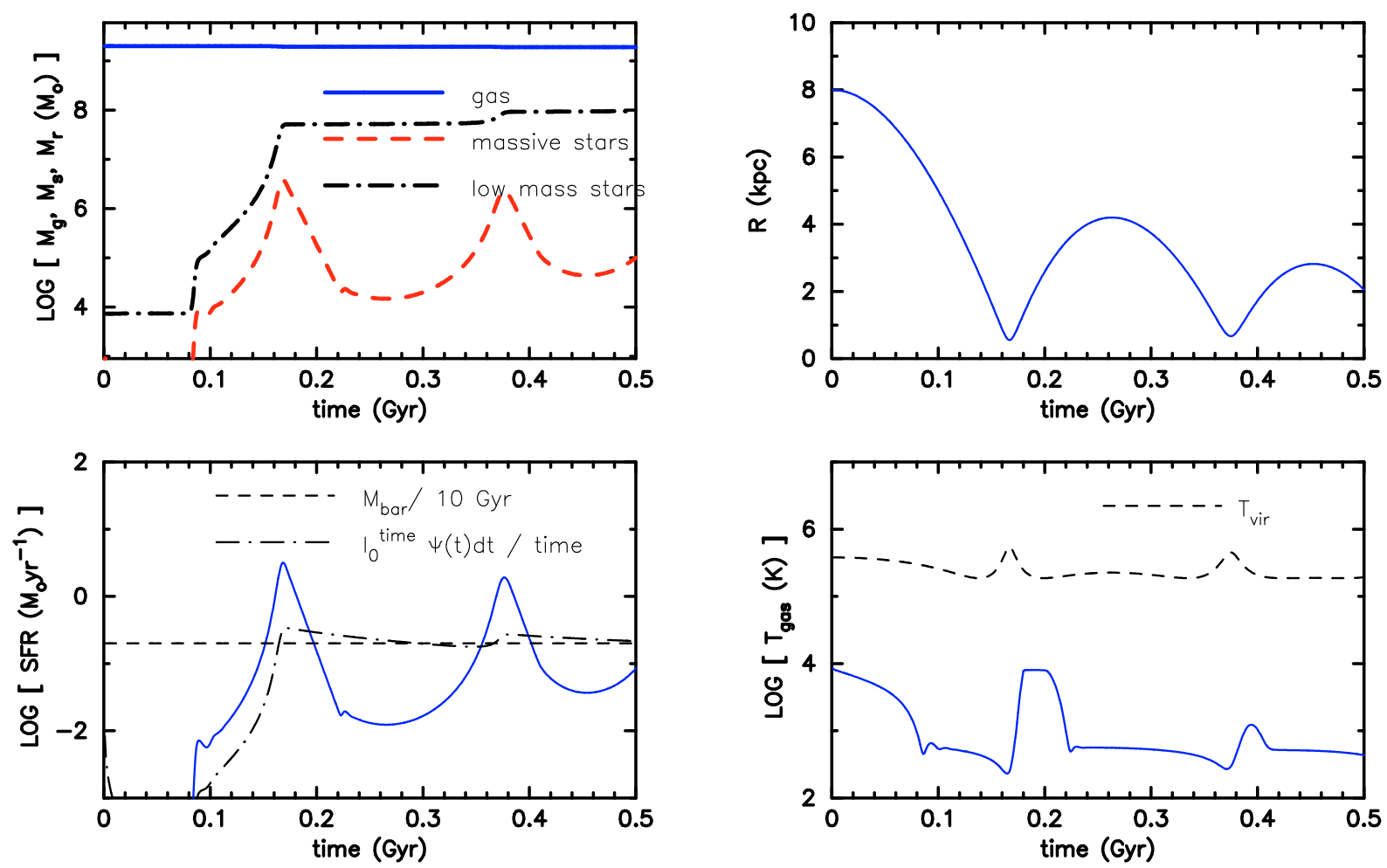

Fig. 2. Initial temporal evolution of a model with a baryonic mass of $2 \times 10^{9} M_{\odot}$. Shown are the masses of the baryonic components (upper left), i.e. gas (solid), low-mass stars, and stellar remnants (dot-dashed), and massive stars (dashed), the mean radius of the system (upper right), the total SFR (lower left), and the gas temperatures (lower right). The SFR is compared with the gas consumption averaged over $10 \mathrm{Gyr}$ and the mean star formation up to time $t$, i.e. $\int_{0}^{t} \Psi\left(t^{\prime}\right) \mathrm{d} t^{\prime} / t$. The temperature is compared with the virial temperature of the system including the self-gravity of the baryons and the dark matter contribution.
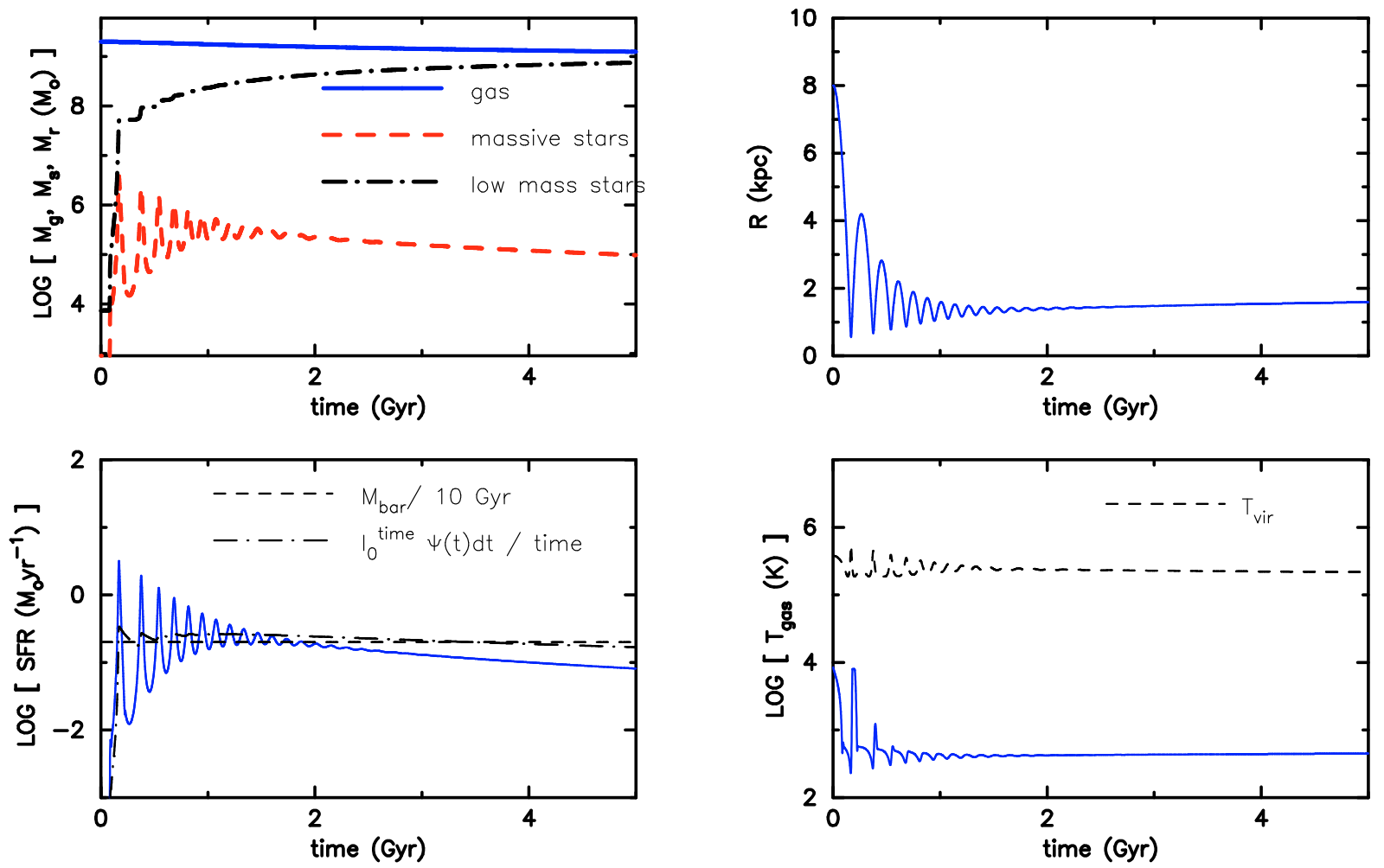

Fig. 3. Same as Fig. 2, but for 5 Gyr of evolution.

temperatures well below the virial temperature. Second, the SFR is strongly modulated by the dynamics. Exceptions to both rules exist, but these are either singular or in physically uninteresting regimes (and beyond the validity of our model equations). 


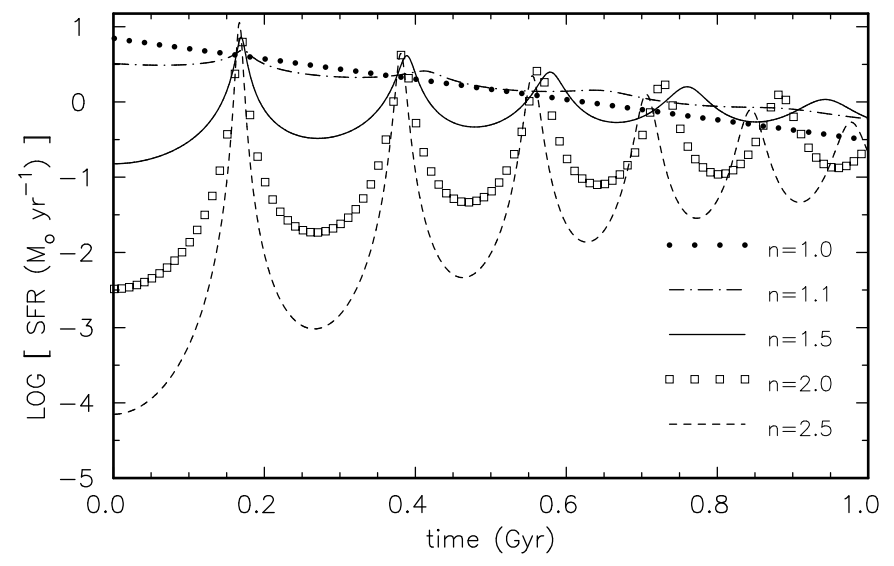

Fig. 4. Temporal evolution of the SFR for models with different exponent in the stellar birth function: $n=1$ (model DN1, filled circles), $n=1.1$ (DN2, dot-dashed), $n=1.5$ (DN3, solid), $n=2$ (DN4, open squares) and $n=2.5$ (DN5, dashed). The normalisation constant $C_{n}$ is kept constant at 0.007 . No negative thermal feedback term is included in the stellar birth function.

\subsection{Influence of stellar birth function}

In this section we address the influence of the stellar birth function and its parametrization according to Eq. (4). In a first series of models (DN1 to DN5), we varied the exponent $n$ in the stellar birth function fixing the constant $C_{n}$ to 0.007 . We also neglected the negative thermal feedback; i.e., we consider the limit $T_{\mathrm{s}} \rightarrow \infty$. This represents the classical Schmidt-law for which the stellar birth function only depends on density, but not on the temperature of the gas.

The Schmidt exponent. Figure 4 shows the temporal evolution of the SFR for different exponents $n$. With increasing $n$, the SFR becomes increasingly sensitive to the density variations. The peaks of the SFR are fairly similar compared to the large SFR variations, whereas the amount of available fuel varies strongly with $n$ as a time integration of the SFR shows: for the linear Schmidt law, it takes 200 Myr to convert $50 \%$ of the gas into stars, whereas for $n=1.5$ this takes about 1.4 Gyr. With increasing nonlinearity of the stellar birth function $\Psi_{b}$, the terms containing the gas density become more important for the SFR and the coupling to the dynamics is reflected in the global SFR. For $n=2$ all oscillations of the SFR within the first $10^{9} \mathrm{yr}$ have amplitudes greater than one order of magnitude.

A linear Schmidt law $(n=1)$ with no thermal feedback term in the stellar birth function represents a singular case with respect to the SFR $\Psi$. For $n=1$, the SFR is proportional to the total gas mass, but independent on the gas density: $\Psi=\Psi_{\mathrm{b}} \cdot V=$ $C_{1} g \cdot V=C_{1} M_{\mathrm{g}}$. This leads to an exponential decay of the gas mass, irrespective of the dynamical state of the system: the virial oscillations do not show up in the exponentially decaying SFR or the amount of massive stars (Fig. 5, left panels).

During the early evolution, the gas temperature is always close to $10^{4} \mathrm{~K}$ (Fig. 5, lower right panel). The high temperatures are a result of stellar heating. Because of a lack of negative thermal feedback in model DN1, the temperature does not regulate the stellar birth function. Only the strong rise in the cooling function beyond $10^{4} \mathrm{~K}$ prevents the gas from reaching higher temperatures. On the other hand, the temperatures are too low in the early stage to become dynamically important as a comparison with the virial temperatures shows (lower right panel in Fig. 5). This lack of star formation induced feedback to the dynamics has already been found in the reference model. Thus, for the linear Schmidt law, both the dynamics and the star formation history are practically decoupled.

The normalisation constant. In a next step we study the influence of the constant $C_{n}$ in the stellar birth function. For a quadratic Schmidt law we varied $C_{n}$ by two orders of magnitude. Initially, the difference in the normalisation constant is directly reflected in the enhanced SFR of model DN6 (Fig. 8). The enhanced SFR leads to a stronger stellar feedback for model DN6. By this, the mean radius increases by about $50 \%$ and the period of the variations is accordingly longer compared to model DN4. The oscillations are more strongly damped and already after about 500 Myr the mean SFR is lower for the model with the larger star formation constant. The latter is a direct consequence of the lack of fuel after the first strong starburst for model DN6.

The negative thermal feedback. So far, we have neglected the thermal feedback $\exp \left(-T / T_{\mathrm{s}}\right)$ in the stellar birth function by setting $T_{\mathrm{s}}=\infty$. When we consider high, but finite, temperature scales $T_{\mathrm{s}}$, the SFR evolves similar to the model without any feedback. Figure 9 compares different $T_{\mathrm{s}}$ values for a linear Schmidt law. In the case of $T_{\mathrm{s}}=10^{5} \mathrm{~K}$ (model DT1) the SFR is almost identic to the case of $T_{\mathrm{s}}=\infty$ (model DN1). After about 1.9 Gyr the SFR suddenly ceases caused by the rapid expansion of the gaseous system when the density drops below a critical density. As discussed in Sect. 4.3, the heating then overcomes the high cooling rates beyond $10^{4} \mathrm{~K}$ and the temperature rises to values above $T_{\mathrm{s}}=10^{5} \mathrm{~K}$ making the feedback term operational.

Reducing $T_{\mathrm{S}}$ to $10^{4} \mathrm{~K}$ (model DT2) brings $T_{\mathrm{S}}$ closer to the actual temperatures of the ISM mainly set by the strong rise in the cooling function. Variations in the temperature influence the stellar birth function much more strongly than in model DT1. This can be seen in the reduced overall SFR, which shows a modulated exponential decay. Setting $T_{\mathrm{s}}$ to $10^{2}$ or $10^{3} \mathrm{~K}$ makes the density variations and its impact on the temperature by cooling to the dominant factor for the global SFR. The oscillation periods are then given by the dynamical timescale and the star formation reaches a quasi-equilibrium state when the virial oscillations have been decayed.

It is also interesting to consider the long-term evolution of model DT2 $\left(T_{\mathrm{s}}=10^{4} \mathrm{~K}\right.$, Fig. 10): after about 6 Gyr of selfregulated quasi-equilibrium evolution, the SFR enters an oscillating period over the next 3 Gyr. Then again the evolution becomes rather stable. This variation of the SFR is directly caused by the temperature fluctuations between about $1.4 \times 10^{4} \mathrm{~K}$ and $8 \times 10^{4} \mathrm{~K}$ as the upper two panels in Fig. 10 show. Since $T_{\mathrm{s}}$ amounts to $10^{4} \mathrm{~K}$, the feedback term varies by about a factor of $10^{3}$. A comparison of the radial variations and the temperature variations shows that the fluctations are not caused by the dynamical evolution (Fig. 10, lower panel): the radial oscillations are too small in amplitude and are not in phase with the temperature variations.

Though e.g. the temperature variations are too large to allow for a complete explanation by linear perturbation theory, a linear stability analysis might give a first clue to the existence of this unstable regime. Since the dynamical evolution is unimportant here, we can apply the results of the stability analysis for the nondynamical set of Eqs. (1)-(3) given in KTH95: a necessary condition for stability is that Eq. (28) of KTH95 holds. In 

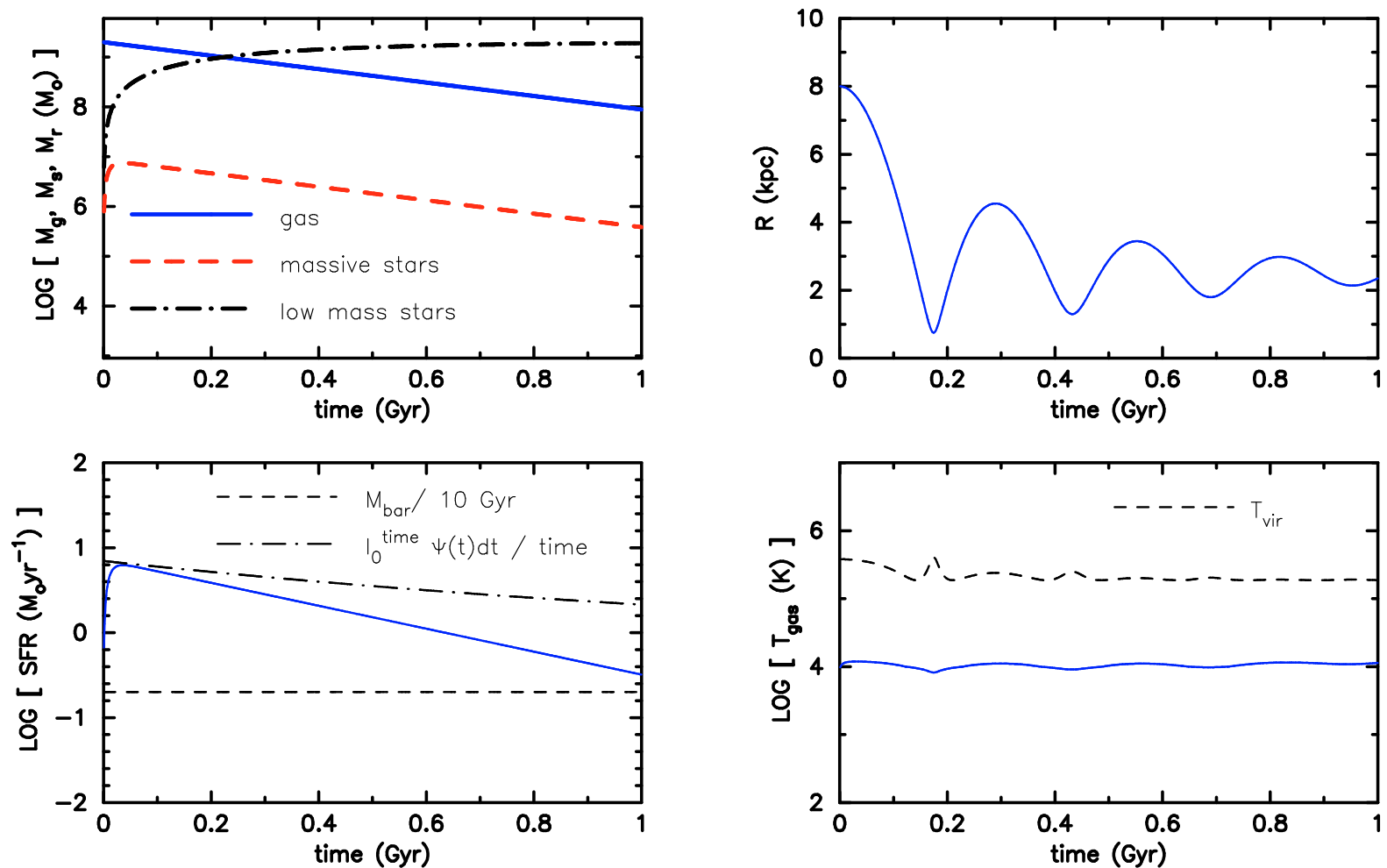

Fig. 5. Temporal evolution of model DN1 characterised by a linear Schmidt law ( $\left.n=1, C_{1}=0.007\right)$, but without a negative thermal feedback term in the stellar birth function. For further details see Fig. 2.
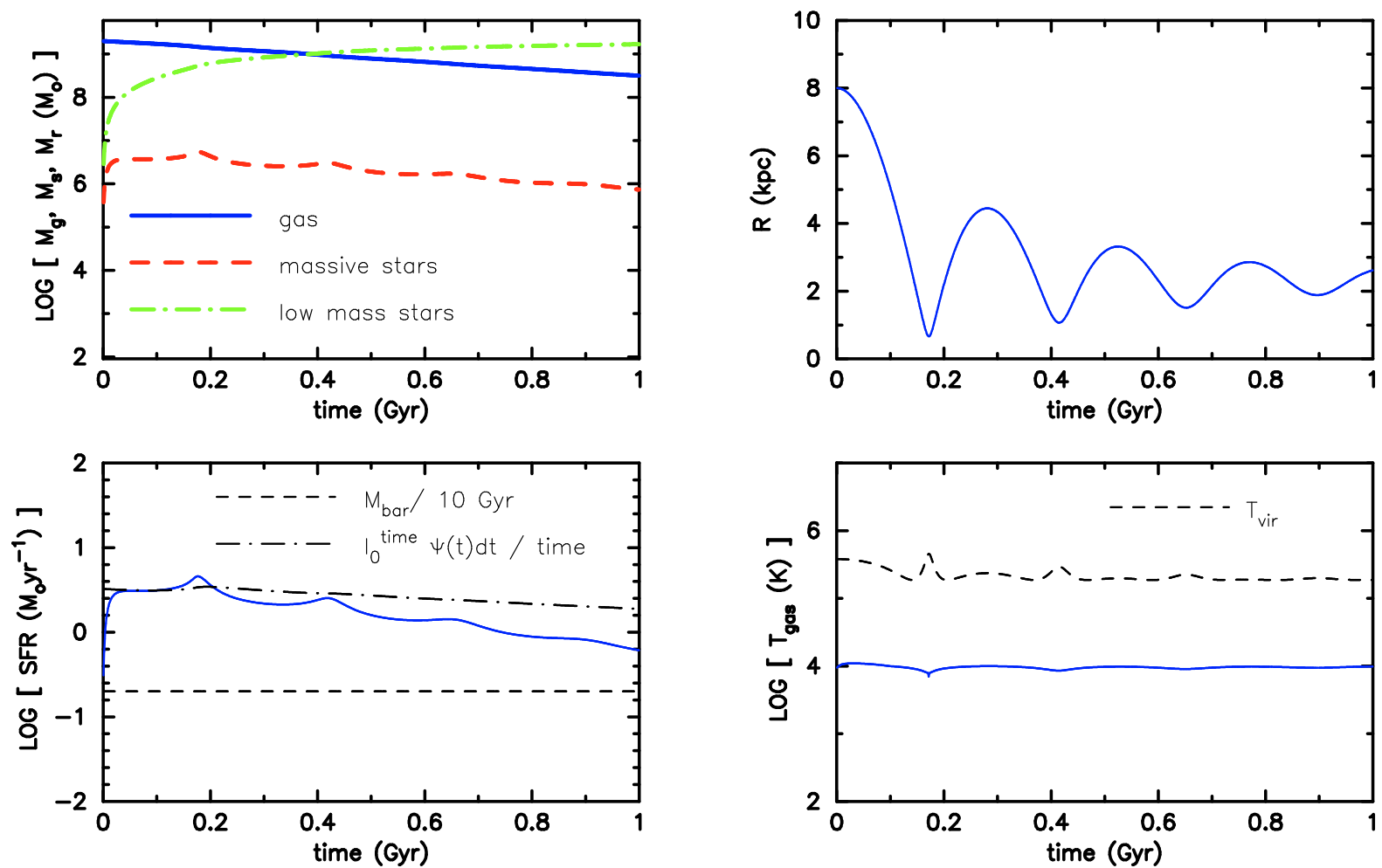

Fig. 6. Temporal evolution of model DN2 characterised by a Schmidt law $\left(n=1.1, C_{n}=0.007\right)$, but without a negative thermal feedback term in the stellar birth function. For further details see Fig. 2.

the limit of low gas densities, we can rewrite this stability criterion as

$\tau_{\text {cool }}>-\Lambda^{\prime}(T) \tau$
This is the case, if the logarithmic slope $\Lambda^{\prime}$ of the cooling function is positive definite (as assumed for the whole temperature regime considered by KTH95). However, the more realistic cooling function applied here drops for temperatures beyond the 

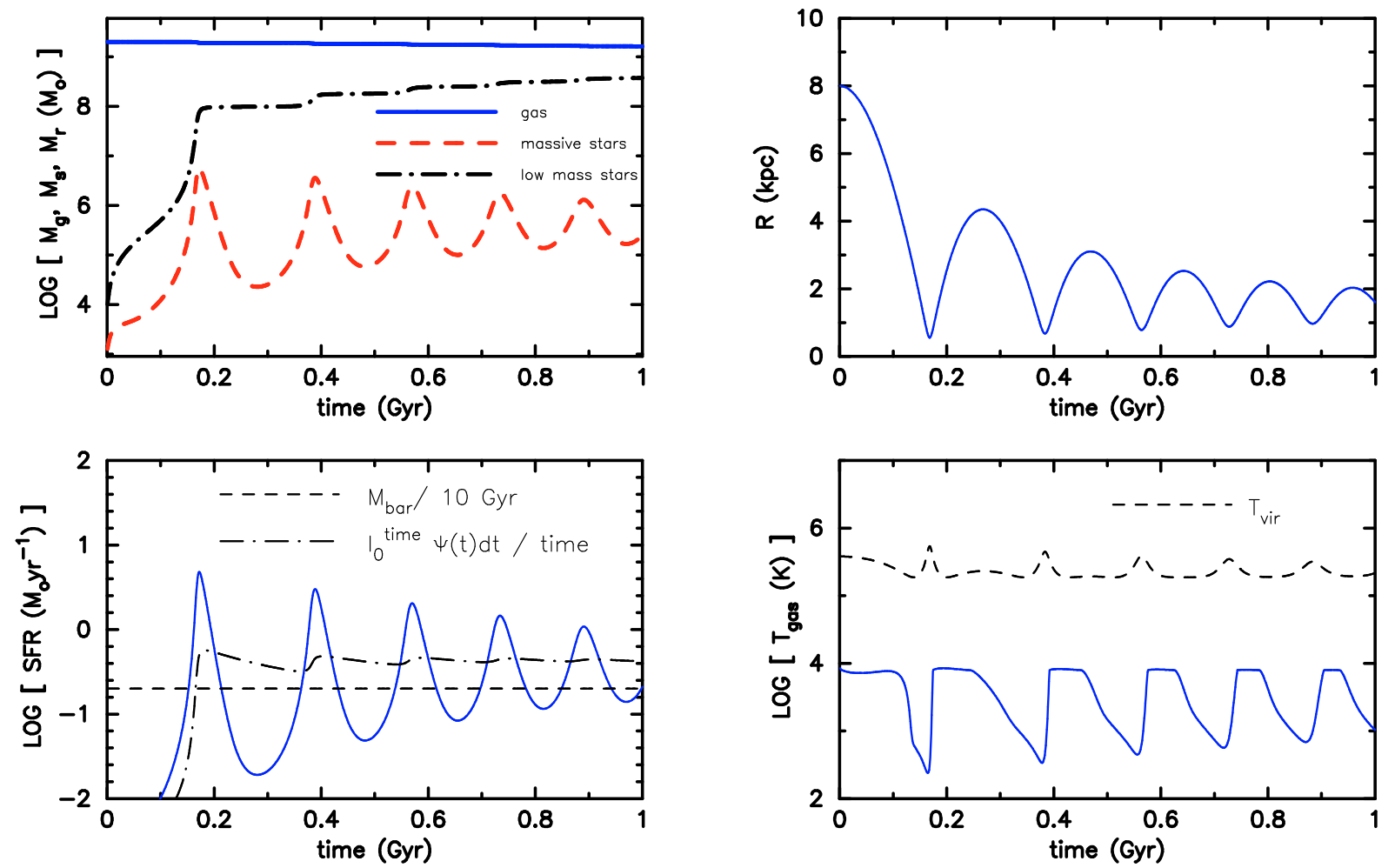

Fig. 7. Temporal evolution of model DN4 characterized by a Schmidt law $\left(n=2, C_{n}=0.007\right)$, but without a negative thermal feedback term in the stellar birth function. For further details see Fig. 2.

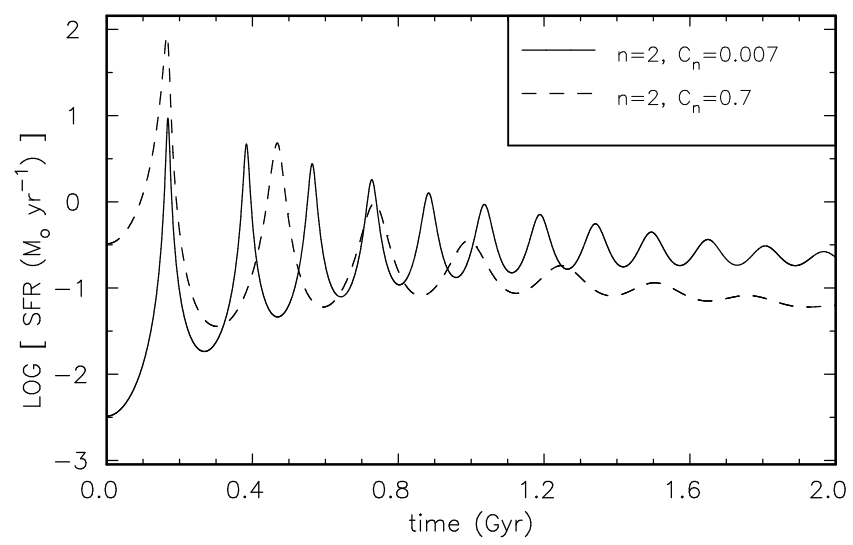

Fig. 8. Temporal evolution of the SFR for the models DN4 and DN6 both characterized by a quadratic Schmidt law with different normalisation constants: $C_{n}=0.007$ (DN4) and $C_{n}=0.7$ (DN6).

peaks caused by hydrogen and helium line emission. Though this behaviour depends on metallicity (becoming weaker with increasing metal content), it persists for metallicities ranging from metal-free to solar metallicity (Böhringer \& Hensler 1989). Thus, the stability of the system is not guaranteed anymore.

Assuming that the logarithmic slope of the cooling function is close to one, instability can only occur if the cooling timescale is longer than the mean stellar lifetime $\tau$ of the massive stars. In the early stages when the gas density is high, the instability criterion will not be met due to the high efficiency of cooling. However, in the late stages when most gas is consumed (or lost), the cooling timescale becomes long even for temperatures above $10^{4} \mathrm{~K}$.

For a metallicity of $1 / 10 Z_{\odot}$, the cooling curve according to Böhringer \& Hensler has a first region of negative slope between

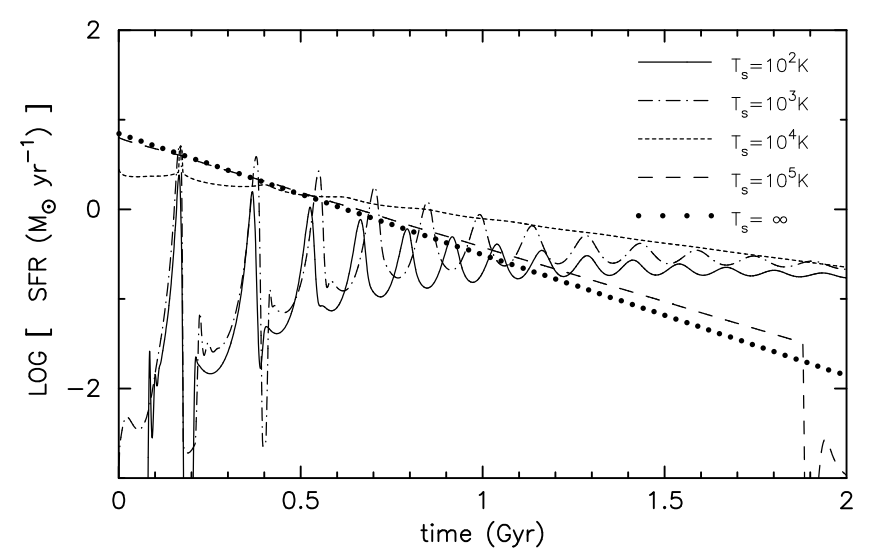

Fig. 9. Temporal evolution of the SFR for models with a linear Schmidt law $(n=1)$ and a negative thermal feedback term with different values of the temperature parameter $T_{\mathrm{s}}: T_{\mathrm{s}}=\infty$ (model DN1, no thermal feedback in the stellar birth function, filled circles), $T_{\mathrm{s}}=10^{2} \mathrm{~K}$ (DT4, solid), $T_{\mathrm{s}}=10^{3} \mathrm{~K}$ (DT3, dot-dashed), $T_{\mathrm{s}}=10^{4} \mathrm{~K}$ (DT2, shortdashed), and $T_{\mathrm{s}}=10^{5} \mathrm{~K}$ (DT1, long-dashed). The normalisation constant $C_{n}$ is kept constant at 0.007 .

$1.5 \times 10^{4} \mathrm{~K}$ and $4 \times 10^{4} \mathrm{~K}$. Therefore, the evolution becomes unstable once the equilibrium temperature rises to $1.5 \times 10^{4} \mathrm{~K}$. The next unstable region caused by He line emission exists for equilibrium temperatures close to $10^{5} \mathrm{~K}$. However, in model DT2 this regime is not reached within the first $10 \mathrm{Gyr}$.

\subsection{Influence of positive stellar feedback}

The star formation recipe discussed in Sect. 3.2 corresponds to a spontaneous star formation mode with a negative stellar feedback. However, there might also be positive stellar 

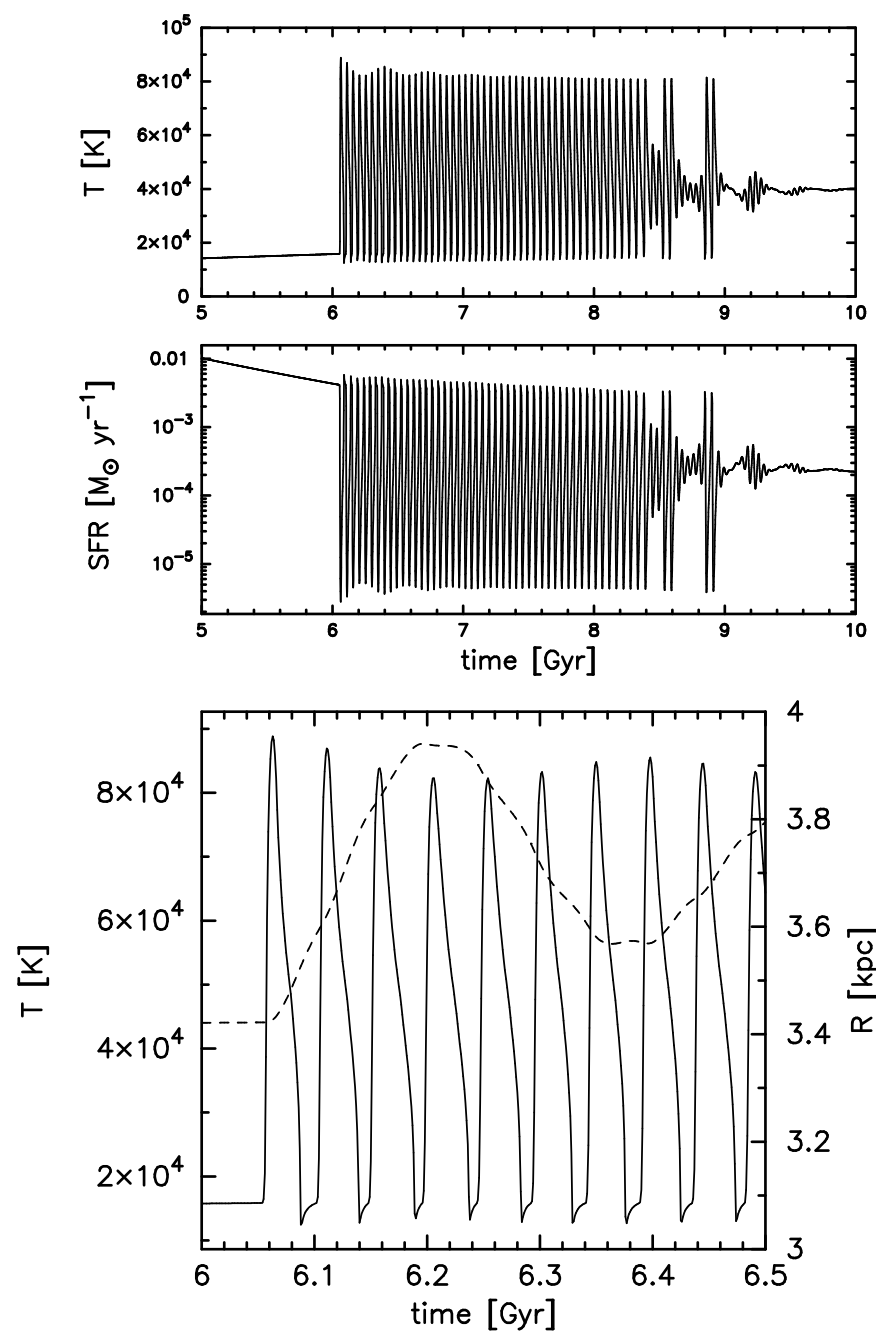

Fig. 10. Late temporal evolution of the gas temperature (upper and lower panel, solid line), the SFR (middle panel) and the mean radius of the system (lower panel, dashed line) for the model with a linear Schmidt law $(n=1)$ and a negative thermal feedback term with $T_{\mathrm{s}}$ (DT2). The lower panel shows only a short fraction of the time displayed in the upper panels.

feedback (see e.g. the stochastically self-propagating star formation models (SSPSF) by Gerola \& Seiden 1978). A model often discussed is the SN-induced star formation (e.g. Ehlerová et al. 1997; Hosokawa \& Inutsuka 2005, 2006): the basic idea is that expanding SN-shells sweep up the ambient ISM in a thin, very dense shell. Such shells cool very efficiently which results in fragmentation and subsequent star formation.

To investigate the influence of an induced star formation mode, we extend our stellar birth function to

$\Psi_{\mathrm{b}}(g, T ; s, R) \equiv \Psi_{\mathrm{b}, \mathrm{sp}}(g, T)+\Psi_{\mathrm{b}, \mathrm{in}}(g, s, R)$,

which allows for both a spontaneous star formation mode and an induced star formation mode. For the spontaneous star formation mode $\Psi_{\mathrm{b}, \mathrm{sp}}(g, T)$ we adopt the description applied in the previous sections; i.e., Eq. (4). For the induced star formation rate we estimate the gas mass swept up by SN shells and turned into stars by

$\Psi_{\mathrm{b}, \text { in }}(g, s, R) \equiv \frac{\eta_{i} g}{\tau_{i}} \cdot f_{i}\left(R_{\mathrm{sh}}(s, g), R\right)$,

where $f_{i}$ denotes the fraction of a galaxy covered by $\mathrm{SN}$ shells, i.e. $g f_{i}$ is the maximum amount of gas undergoing
SN shell-induced star formation. A fraction $\eta_{i}$ of this mass is then converted on a timescale $\tau_{i}$ into stars. The factor $f_{i}$ is related to the volume $R_{\mathrm{sh}}^{3}$ filled by massive superbubbles undergoing fragmentation relative to the volume of the galaxy. For simplicity we assume

$f_{i}\left(R_{\mathrm{sh}}(s, g), R\right) \equiv 1-\mathrm{e}^{-\left(R_{\mathrm{sh}} / R\right)^{3}}$.

In the case of low star formation activity, only a small fraction of the galactic volume is affected by induced star formation, whereas for high star formation activity most of the gas mass might be swept up. The related star formation timescale $\tau_{i}$ is given by the time for bubble creation. The efficieny factor $\eta_{i}$ gives the fraction of gas accumulated in shells that is converted into stars. We used a value of $\eta_{i}=0.1$ here. The size and the timescale for the bubble evolution were taken from Eqs. (13) and (14) of Ehlerová et al. (1997). It should be noted that this star formation recipe is only meant to be a simple qualitative estimate, not a detailed sophisticated induced star formation model. Anyway, we believe that this ansatz covers all essential ingredients for the SN-induced star formation mode.

Figure 11 displays the SFR for different combinations of the star formation modes. In case of a low temperature $T_{\mathrm{s}}=100 \mathrm{~K}$, the overall SFR is dominated by the induced star formation (ISF) mode (upper panel of Fig. 11) as the agreement between model DI1 (ISF only mode) and the model DI2 (combined star formation modes) demonstrates. This is caused by the high temperatures of about $10^{4} \mathrm{~K}$ created by the massive stars formed by induced star formation. Due to negative thermal feedback the spontaneous star formation is almost completely suppressed. This suppression can, of course, not take place in a purely spontaneous star formation model (model A). In that case, the amplitudes of the density driven SFR variations increase because of the temperature dependent variations in the feedback term. The dominance of the dynamical evolution is reflected in the almost identical periods of the SFR in all models. The slightly longer periods for the models DI1 and DI2 (which include induced star formation) stem from the larger equilibrium radii leading to a longer free-fall time.

To have a substantial contribution from the spontaneous star formation mode in a stellar birth function including induced star formation, the high efficiency of the thermal feedback has to be reduced. This can be realized by an increase in the feedback temperature scale $T_{\mathrm{s}}$ close to or above the actual gas temperature. Model DI3 has a $T_{\mathrm{s}}$ of $10^{4} \mathrm{~K}$ : models using either of the two star formation modes (induced SF, spontaneous SF) or both of them become then comparable in star formation amplitudes, peak values and the overall production rate of stars (Fig. 11, lower panel). Still, the SFR is dominated by the dynamically driven oscillations. It is interesting to note that after $1 \mathrm{Gyr}$ the combined SFR (model DI3) becomes smaller than both singlemode models (DI1 and A2).

The relative fraction of the spontaneous and the induced star formation mode in the combined model DI3 with respect to the total number of stars formed is shown in Fig. 12: at the very beginning the spontaneous star formation exceeds the induced star formation. However, with an increasing overall SFR the induced star formation begins to dominate and the spontaneous star formation drops to $30 \%$. The contribution of the induced star formation drops already before reaching the first peak of the SFR: this is caused by the dynamics, i.e. the increase in the gas density during the collapse of the system and the subsequent enhanced spontaneous star formation. The temperature of the gas remains almost constant close to $10^{4} \mathrm{~K}$ during this evolution. 

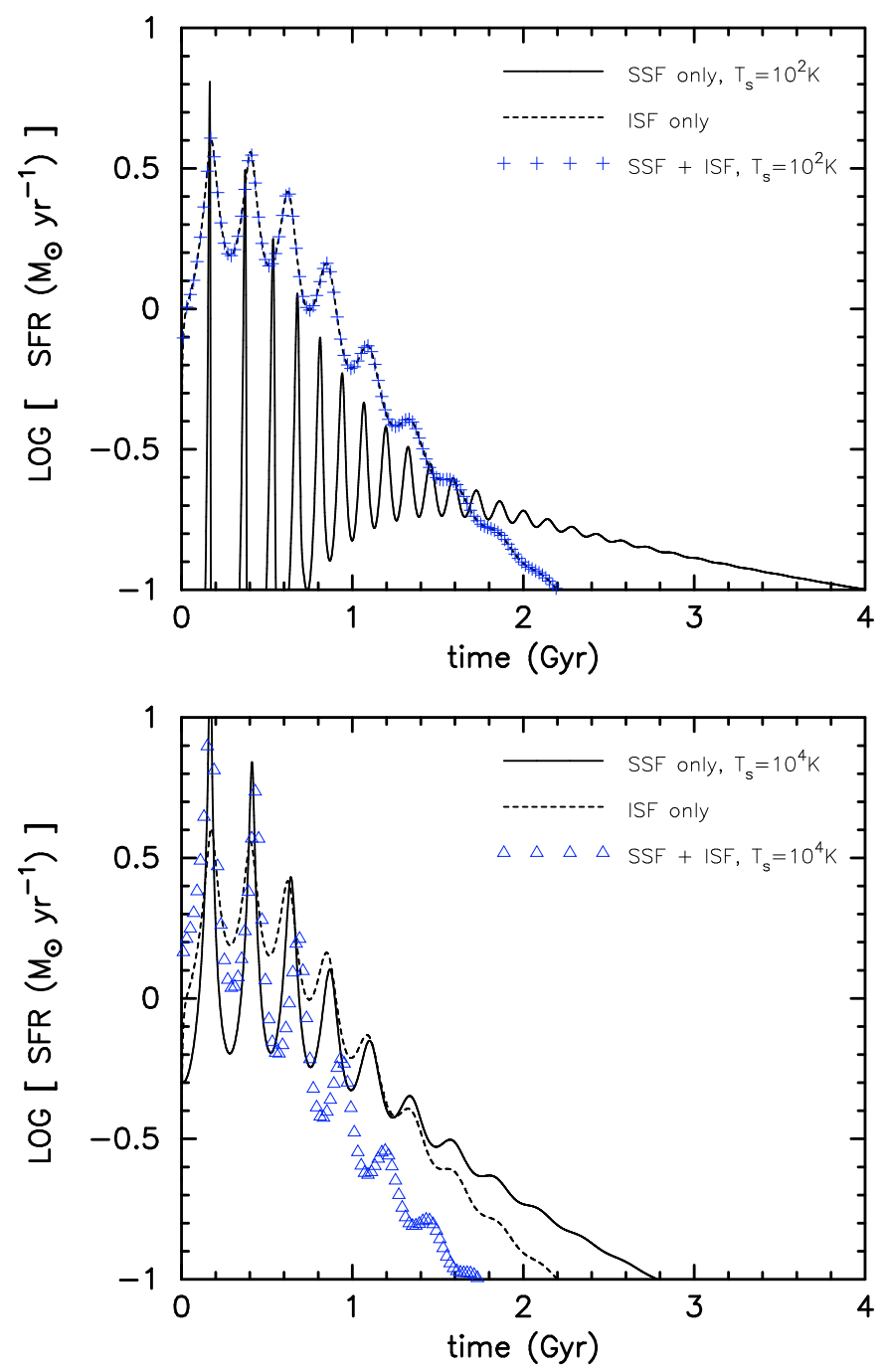

Fig. 11. Temporal evolution of the SFR for models with different stellar birth modes: induced star formation only (dashed line, DI1), spontaneous star formation only (solid line; $T_{\mathrm{s}}=100 \mathrm{~K}$ (model A, upper panel) and $T_{\mathrm{s}}=10^{4} \mathrm{~K}(\mathrm{~A} 2$, lower panel)) and both star formation modes together $\left(T_{\mathrm{s}}=100 \mathrm{~K}\right.$ (DI2, plus signs in upper panel) and $T_{\mathrm{s}}=10^{4} \mathrm{~K}(\mathrm{DI} 3$, open triangles in lower panel $)$ ).

After a Hubble time about $62 \%$ of the stars are produced by induced star formation.

It is remarkable that adding induced star formation does not (necessarily) result in a quick consumption of all available gas. For our fiducial model, roughly half of the stars are formed in one of the two star formation modes. Though this fraction can be varied by e.g. amplifying the thermal feedback term of the spontaneous star formation mode or changing the efficiency factor $\eta_{i}$ of the induced star formation, none of the star formation modes leads to a completely different behaviour of the system: still the overall star formation rate is controlled by the global dynamics.

\subsection{Influence of the IMF}

Constant IMF. A variation in the stellar initial mass function will mainly affect the mass fraction $\xi$ of massive stars. To test limiting cases we varied the time-independent $\xi$ by a factor of 2 . Though such a change is practically beyond the limits of generally adopted standard IMFs, the impact on the evolution is small;

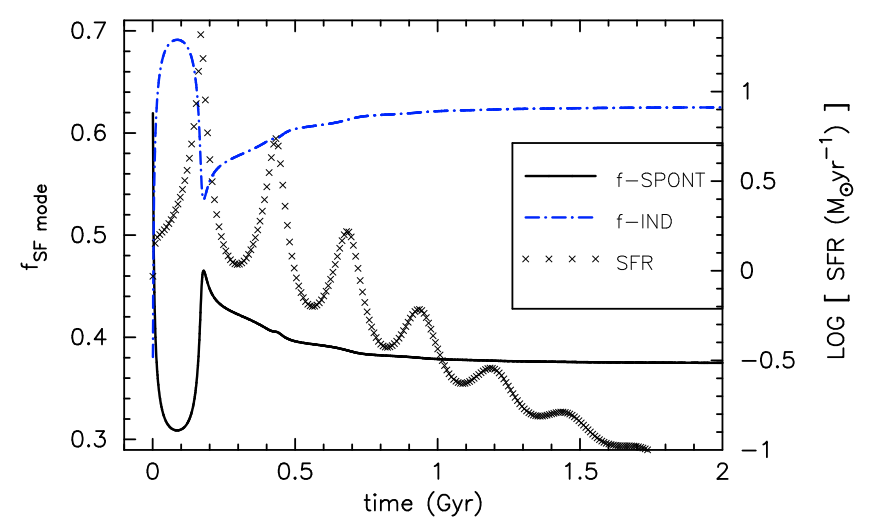

Fig. 12. Temporal evolution of the relative contribution of the spontaneous (solid) and the induced (dot-dashed) star formation mode to the total mass converted into stars for model DI3. For comparison the combined SFR is also shown (plus).

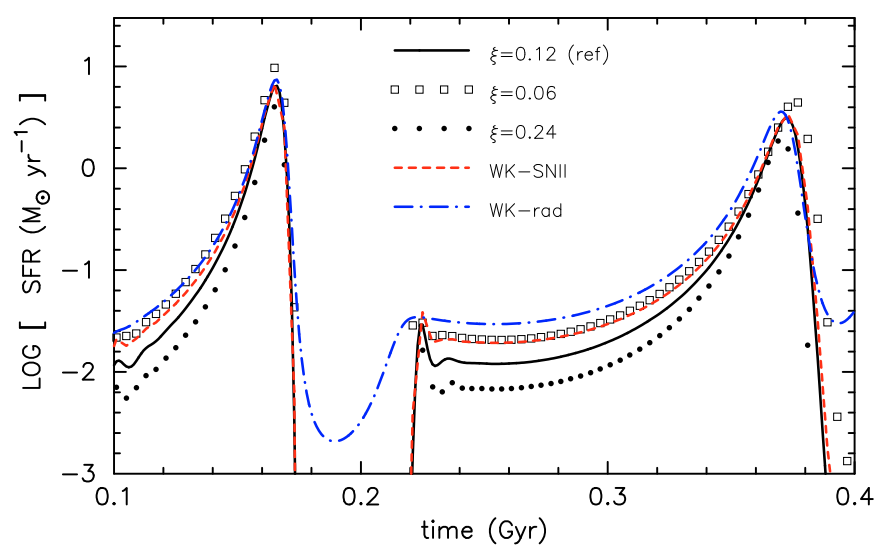

Fig. 13. Temporal evolution of the SFR for the reference model A (solid), models DX1 and DX2 with a constant IMF-derived fraction $\xi$ of massive stars ( $\xi=0.06$ : open squares; $\xi=0.24$ : filled circles) and the Weidner-Kroupa type variable IMF models based on SNII heating (DWK1, dashed) and radiative heating (DWK2, dot-dashed).

e.g. the SFR is qualitatively identical to the reference model for both $\xi$-values (Fig. 13). The small quantitative differences (e.g. lower SFR for larger $\xi$ ) can be understood as a result of enhanced $(\xi=0.24)$ or reduced thermal feedback. The weak response of the SFR (and especially its peak values) is a result of the strong self-regulated coupling of the star-gas system. As a result, the evolution of the star-gas system is practically insensitive to reasonable variations in the constant IMF.

Variable IMF. Recently, Weidner \& Kroupa (2005) suggested a variable IMF that depends on the global star formation activity of the galaxy. Their main idea is that relations exist between the global galactic SFR and the maximum mass of the molecular clouds as well as between the cloud mass and the maximum mass of the most massive stars in star formation regions. By this, the global SFR affects the IMF for massive stars which are responsible for the main heating and the metal production.

To investigate such a scenario we modified our equations by introducing an efficiency factor $f_{\mathrm{WK}}(\Psi)$ for the production of massive stars; i.e., we replaced $\xi$ by $\xi \cdot f_{\mathrm{WK}}(\Psi)$. The efficiency 
factor was given by a simple fitting formula adopted from the analysis of Köppen et al. (2006):

$f_{\mathrm{WK}}=\left\{\begin{array}{ccc}1-0.8 \cdot \mathrm{e}^{-x / 2} & & x \geq 0 \\ 0.2 \mathrm{e}^{x} & \text { for } & \\ & & x<0\end{array}\right.$

with $x \equiv 3+\log \left[\Psi /\left(M_{\odot} \mathrm{yr}^{-1}\right)\right]$. One should note that the efficiency factor only varies slightly over the physically interesting regime of $x>0$ or $\Psi>10^{-3} M_{\odot} \mathrm{yr}^{-1}$.

We considered two cases for the energy feedback. In the first case we assumed that the feedback is given by type II supernovae (model DWK1). In that case the heating coefficient corresponding to the energy input per supernova has to be multiplied by the number of massive stars. The number of massive stars, however, depends mainly on the lower mass limit of the mass range of massive stars, and not on the upper mass region that is mainly affected by a Weidner-Kroupa type IMF. A detailed calculation shows that the conversion factor between the number of massive stars and their mass varies only by a factor of 2 when changing the SFR from $10^{-3} M_{\odot} \mathrm{yr}^{-1}$ to $100 M_{\odot} \mathrm{yr}^{-1}$. In our model DWK1 we neglect this small variation and keep the energy equation.

In the second case, we assume that the heating is mainly done via radiation (model DWK2). Then, the heating coefficient $h$ becomes a function of the SFR, because of the strong dependence of the stellar radiative energy input on the upper stellar masses caused by the mass-luminosity relation. A detailed calculation shows that the corresponding efficiency factor is similar to the one for the mass fraction. Therefore, we use for simplicity the same factor $f_{\mathrm{WK}}$, i.e. $h$ is replaced by $h \cdot f_{\mathrm{WK}}(\Psi)$ in Eq. (11) (or Eq. (3), respectively).

Figure 13 shows the evolution of both models. The difference between models with and without a variable IMF are marginal in both, amplitude and timing. Compared to the reference model A, the Weidner-Kroupa-type IMF models have a slightly enhanced SFR. This enhancement is necessary to balance the deficit in the stellar feedback caused by the reduced number of massive stars in the Weidner-Kroupa-type IMF models.

In general the qualitative evolution of the models is not affected by the shown IMF variations, either if we change the mass fraction $\xi$ assuming a stationary IMF or if we apply the temporal variable star formation-dependent IMF suggested by Weidner \& Kroupa (2005). The main reason is the very efficient self-regulation.

\section{Discussion}

\subsection{Timescales}

Basically four physically distinct timescales are involved in our description: the heating and cooling timescales, $\tau_{\mathrm{fb}}$ and $\tau_{\text {diss }}$, the dynamical timescale $\tau_{\text {dyn }}$ and the gas consumption (or star formation) timescale $\tau_{\mathrm{SF}}$. KTH95 already has shown that $\tau_{\mathrm{SF}}$ is usually longer than the timescales governing the energetics of the ISM. This also holds in case of a multiphase ISM model (Köppen et al. 1998), where the timescale for the additional equilibrium between evaporation and condensation of the gas clouds is well separated from the other two, leaving them unperturbed. Therefore, we expect that inclusion of a multiphase ISM model would not alter the basic behaviour of our system.

For typical mean gas densities the dynamical timescale exceeds also the cooling and heating time (though it is less than the gas consumption time). Therefore, the system almost instantaneously adjusts to the equilibrium state given by the actual gas density. Provided the temperature remains in a regime with a positive slope of the cooling function, the system is then stable. This holds e.g. for temperatures below $10^{4} \mathrm{~K}$, (which is practically the temperature regime for high and moderate gas densities). Thus, the radial oscillations are only initial transient virial oscillations acting on a dynamical timescale.

This behaviour turned out to be rather robust against variations in model parameters like stellar heating, gas mass, initial spatial extension, stellar birth function (with the exception of a linear stellar birth function resulting in an exponentially decaying SFR). Also different IMF parametrizations or even a variable IMF (like the integrated galactic IMF suggested by Weidner \& Kroupa 2005) do not change the behaviour of the system qualitatively provided the feedback mechanisms are not cutoff.

\subsection{Generic behaviour}

Our calculations show that the evolution of the coupled stargas system is dominated by the dynamical state of the system. Due to the short heating and cooling timescales, the ISM relaxes quickly. Thus, the system's behaviour is governed by the dynamical equation Eq. (7), which has the structural form

$\frac{\mathrm{d} R}{\mathrm{~d} t}=v_{\mathrm{rad}}$

$\frac{\mathrm{d} v_{\mathrm{rad}}}{\mathrm{d} t}=-\frac{A_{2}}{R^{2}}+\frac{A_{1}}{R}+\frac{A_{3}}{R^{3}}-A_{4} v_{\mathrm{rad}}$

with coefficients $A_{i}$ that are positive for physically sensible descriptions. For simplicity we only consider the self-gravity term here. With a proper choice of the time unit, $A_{4}$ can be set to unity.

For $v_{\text {rad }}=0$ there exist equilibrium positions, where the forces cancel out

$\frac{A_{1}}{R_{\mathrm{e}}}-\frac{A_{2}}{R_{\mathrm{e}}^{2}}+\frac{A_{3}}{R_{\mathrm{e}}^{3}}=0$

or

$A_{1} R_{\mathrm{e}}^{2}-A_{2} R_{\mathrm{e}}+A_{3}=0$

with the formal solution

$R_{\mathrm{e} \pm}=\frac{1}{2 A_{1}}\left(A_{2} \pm \sqrt{A_{2}^{2}-4 A_{1} A_{3}}\right)$.

If $A_{2}^{2}>4 A_{1} A_{3}$, two real equilibrium radii exist. Note that for $A_{1}=0$ (i.e. a gas temperature well below the virial temperature) one simply has a single solution

$R_{\mathrm{e}}=\frac{A_{3}}{A_{2}}$

The analysis of the local stability of these equilibrium points (via the linearized equations) of the Jacobi matrix $\mathbf{J}$

$\frac{\partial \dot{R}}{\partial R}=0$

$\frac{\partial \dot{R}}{\partial v_{\mathrm{rad}}}=1$

$\frac{\partial \dot{v}_{\mathrm{rad}}}{\partial R}=-\frac{A_{1}}{R_{\mathrm{e}}^{2}}+\frac{2 A_{2}}{R_{\mathrm{e}}^{3}}-\frac{3 A_{3}}{R_{\mathrm{e}}^{4}}$

$\frac{\partial \dot{v}_{\mathrm{rad}}}{\partial v_{\mathrm{rad}}}=-1$ 
with

Trace $\mathbf{J}=-1$

$\operatorname{det} \mathbf{J}=\frac{A_{1}}{R_{\mathrm{e}}^{2}}-\frac{2 A_{2}}{R_{\mathrm{e}}^{3}}+\frac{3 A_{3}}{R_{\mathrm{e}}^{4}}$

Discr $=1-4 \operatorname{det} \mathbf{J} /(\text { Trace } \mathbf{J})^{2}$

shows if equilibrium solutions exist $\left(A_{2}^{2}>4 A_{1} A_{3}\right)$ that $R_{\mathrm{e}-}$ always is an attracting focus, since the trace and the discriminant are negative for all positive coefficients $A_{k}$. Likewise, the other point $R_{\mathrm{e}+}$ always is a saddle point (negative determinant).

The consequences of this general structure are shown by a few representative trajectories in the phase space. In Fig. 14 we depict these already for the complete system of our equations. One notes that if the initial conditions are close enough to the attracting focus, the system performs damped nonlinear oscillations before settling in the equilibrium. If the initial radius or the initial speed is too large, there will be a collapse, followed by an expansion. This takes the system to the vicinity of the saddle point, whence the expansion continues without bounds.

If one looks more closely at the behaviour in the equilibrium state (shown in the inset in Fig. 14), one finds that the equilibrium radius does not stay constant, but always increases, more or less rapidly depending on the coefficients $A_{i}$. The reason is that the coefficient $A_{1}$ of the pressure term in the full set of equations depends on the gas temperature. When the system reaches the (dynamical) equilibrium, the gas continues to be consumed by star formation. The cooling of the gas therefore slows down, and the gas temperature increases slowly, giving rise to a slow expansion that contributes to the decrease in the gas density. This rise in temperature causes an increase in the equilibrium radius, which the system simply follows. This leads to the slow drift seen in the inset of Fig. 14.

In Fig. 15 we plot a trajectory in the radius-temperature plane. One notes that the oscillations in radius and temperature go with a nearly constant phase lag, but once the oscillations have damped out, a steady secular increase in both radius and temperature takes over (cf. to inset in Fig. 15).

\subsection{Heating-cooling equilibrium at low gas densities}

The equilibrium between heating and cooling plays a crucial role for the overall evolution of our system. Cooling is very efficient for typical galactic mean densities, thereby compensating for the stellar heating processes. However, when the gas density drops, the heating by stars $\left(h M_{\mathrm{s}} \propto h \Psi_{\mathrm{b}} V \propto M_{\mathrm{g}}\right)$ can overcome the cooling $\left(\Lambda g^{2} V \propto g M_{\mathrm{g}}\right)$, so the gas quickly heats up (here we assume a linear stellar birth function). Once temperatures become higher than the virial temperature, the gaseous system expands and a mass loss occurs even without any dramatic event like a starburst. The critical density $g_{\text {loss }}$ for gas loss can be estimated from the balance between heating and cooling from Eq. (3) with $s \approx \zeta \Psi \tau=\zeta C_{n} g_{\mathrm{loss}}^{n} \mathrm{e}^{-T / T_{\mathrm{s}}}$

$g_{\mathrm{loss}}^{2-n}=\frac{h \zeta C_{n} \tau \mathrm{e}^{-T / T_{\mathrm{s}}}}{\Lambda(T)}$.

For the values of model DN1 (linear Schmidt law), we get $g_{\text {loss }} \sim 8 \times 10^{-5} M_{\odot} \mathrm{pc}^{-3}$ by adopting a cooling rate of $\Lambda \approx$ $10^{-21.5} \mathrm{erg} \mathrm{cm}^{3} \mathrm{~s}^{-1}$ characteristic of temperatures well above the drop near $10^{4} \mathrm{~K}$. The $g_{\text {loss }}$ becomes smaller with increasing Schmidt exponent $n$. Practically, such low gas densities might not be relevant, e.g., because of the gas replenishment by lowmass stars.

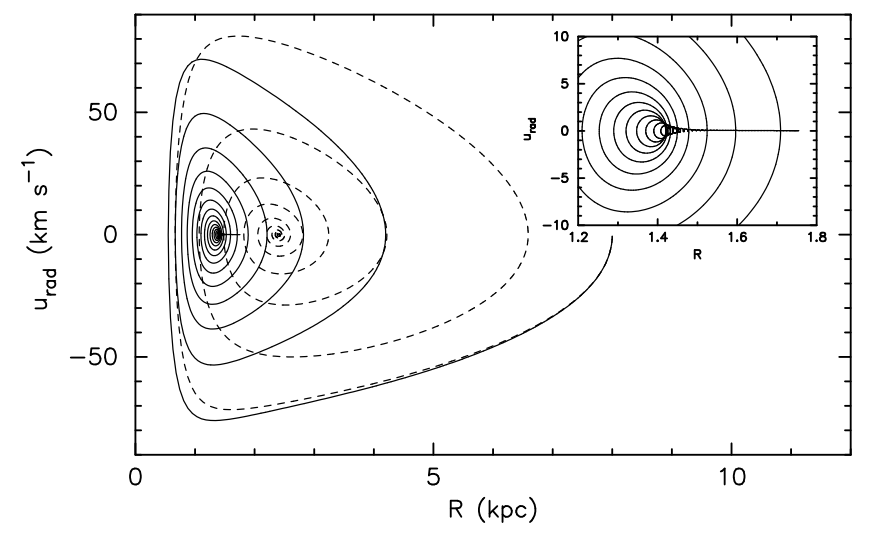

Fig. 14. Evolution of the reference model A (solid) and the model DT5 (quadratic Schmidt law with feedback term, $T_{\mathrm{s}}=10^{5} \mathrm{~K}$; dashed line) in the phase space $R-v_{\text {rad }}$. The inset shows a blow-up of model A near its equilibrium position reached after the initial oscillations.

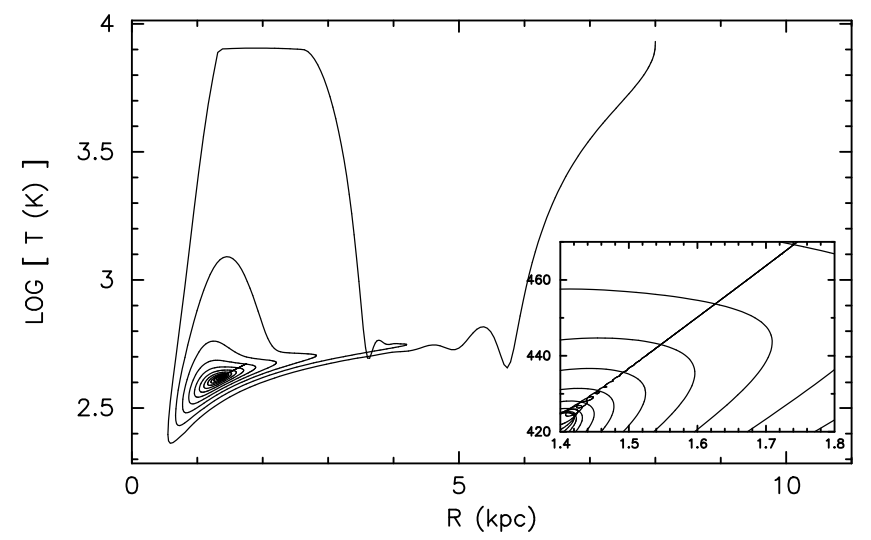

Fig. 15. Evolution of the reference model $\mathrm{A}$ in the $R-T$ plane. The inset shows a blow-up near the equilibrium position reached after the initial oscillations. In the inset linear temperature values are used.

It is interesting to note that no critical density exists for a quadratic Schmidt law. If $n$ is higher than 2, the system would even run into a cooling catastrophe. In the case of optically thin heating, $h$ in Eq. (34) has to be replaced by $\tilde{h} g$. Thus, the critical exponent for a cooling catastrophe already becomes $n=1$. This means that, for all reasonable stellar birth functions, the gas cools down below a critical density. In that case other heating mechanisms providing a steady energy source independent of the amount of massive stars (hence, the SFR and the gas density) might become significant.

\subsection{Are the dynamics influenced by stellar feedback?}

In this paragraph we discuss the impact of stellar feedback on the dynamical evolution. The latter is described by Eq. (7) which contains a pressure gradient term: $1 / g \cdot \mathrm{d} P / \mathrm{d} r \sim T / R$. Stellar feedback can influence the dynamical evolution via this term by heating. Practically speaking, this happens when the pressure term is close to or exceeds the gravitational acceleration terms (the first two terms in Eq. (7)), i.e. when the gas temperature is close to the virial temperature or higher. However, in case of the evolutionary stages shown in this paper the gas temperatures are always of the order $10^{4} \mathrm{~K}$ or below, whereas the virial temperature is at least a few $10^{5} \mathrm{~K}$. Physically, the low gas temperatures are caused by the steep rise in the cooling function beyond $10^{4} \mathrm{~K}$ (cf. e.g. Fig. 1 in Böhringer \& Hensler 1989). For typical gas 


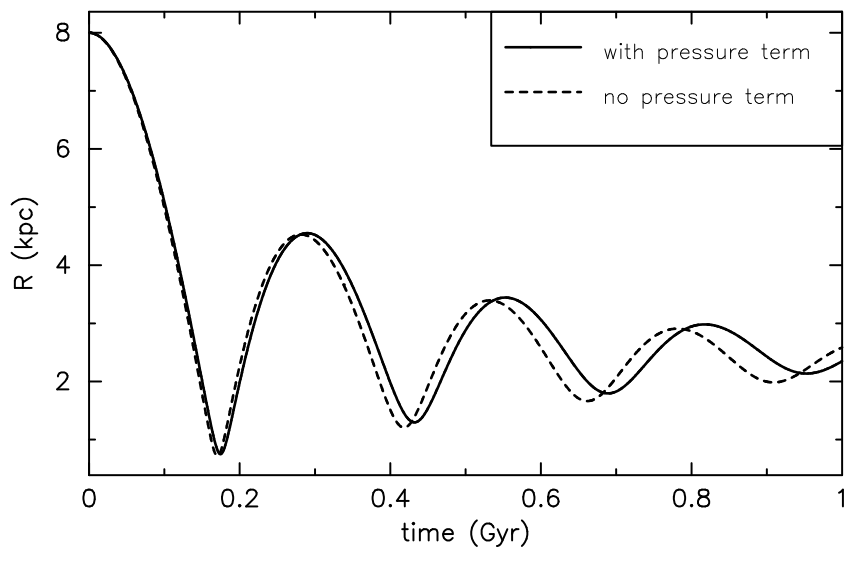

Fig. 16. Temporal evolution of the mean radius of model DN1 (solid) and the same model, but neglecting the pressure term in the dynamical equation Eq. (7) (dashed).

densities, unrealistically high heating rates are required to reach (and keep) temperatures beyond $10^{4} \mathrm{~K}$.

Therefore, the dynamics are almost unaffected by the thermal state of the gas. This is nicely illustrated in Fig. 16 where the mean radius of model DN1 is compared with a model neglecting the pressure term of Eq. (7): no substantial difference between both models is discernible. Small differences develop at maximum compression when the gravitational terms and the angular momentum almost cancel. For this short moment, the small pressure-gradient term contributes recognisably. It acts as a reduction of the gravity, e.g. like a density reduction, which results in slightly longer periods.

The situation only changes when either the gas temperature rises or the virial temperature decreases. The latter can happen for systems of lower mass, e.g., for compact systems like globular clusters (no DM, $\sim 10^{6} M_{\odot}$ ) or more extended systems like $\mathrm{dSph}$ galaxies, when the virial temperatures drop down to a few $10^{4} \mathrm{~K}$ (we will discuss this in a forthcoming paper). Alternatively, the gas temperature might be heated to a few $10^{5} \mathrm{~K}$. Practically speaking, this is only the case where the criterion for run-away heating, Eq. (34), is fulfilled, i.e. when the gas density drops below a critical value due to gas consumption. The actual critical values, however, are too low, to be practically relevant within the limits of our model.

The physical reason for the general insensitivity of the dynamics to the thermal feedback induced by stars is the steep rise in the cooling function near $10^{4} \mathrm{~K}$. As long as radiative cooling is the main agent of energy dissipation and as long as the gas temperature is kept below $10^{4} \mathrm{~K}$, thermal feedback will only affect the dynamics of systems with a virial temperature near $10^{4} \mathrm{~K}$. However, for short periods (especially at early stages), the gas temperature might reach or exceed temperatures of $10^{5} \mathrm{~K}$ briefly affecting the dynamics of systems with virial temperatures of that order.

However, thermal pressure (and therefore classical cooling) does not need to be the main agent counter acting gravity. If the system is composed of sufficiently compact objects, their velocity dispersion will provide a kinetic pressure, e.g., in case of a cloud system, the internal temperature of the clouds is irrelevant for their overall dynamics, which is driven by the velocity dispersion. In that case, inelastic cloud-cloud collisions provide the relevant energy dissipation mechanism. Different from radiative cooling, there is no steep increase in the cloud-cloud "cooling" function and the arguments preventing a strong mutual coupling between dynamics, star formation, and stellar feedback are not applicable. Additionally, the timescale for energy dissipation due to cloud-cloud collisions is much longer than the radiative cooling timescales, which also might allow for more of a variety of responses by the system. We will discuss the related aspects in a future paper.

\subsection{Comparison with "full" dwarf galaxy simulations}

How do our simple models compare with detailed numerical simulations? Though important, it is a difficult question. At the moment there are only a small number of sophisticated simulations available. They differ by many aspects between themselves and from our calculations: e.g. with respect to the implemented physics, the numerical methods or the initial conditions and the general physical set-up. Unfortunately, a standard test for dwarf galaxy evolution/physics does not exist.

With all these differences in mind a detailed comparison is not meaningful, but a qualitative comparison should shed light on the applicability of our ansatz; e.g., Pelupessy et al. (2004, hereafter PWI04) model the evolution of a rotating disklike dwarf galaxy embedded in a dark matter halo. They apply a sophisticated ISM model including heating, cooling and an ionisation/recombination scheme. Their SFR develops a quasiperiodic behaviour with a period of about twice the dynamical time for (weak) bursts. PWI04 interpret this behaviour as the result of a kick that drives the gas out of the disk and the subsequent fallback of the gas initiating the next star formation episode. This concept is very similar to our adopted coupling between feedback and dynamics, though we are investigating a spherical system.

The overall star formation peaks in PWI04 are much smaller than in our calculations (by one order for a fiducial model including a feedback term). This might be caused by the different initial set-ups, because the spherical configuration we study allows for a much deeper collapse than a rotationally supported disk. As a result, the density and, thus, the star formation variations are much higher in our case than for PWI04. For comparison, the full 1d chemo-dynamical models of Hensler et al. (2004, afterwards HTG04) model spherical galaxies (but without dark matter) and they exhibit SFR comparable to ours. Similar to our calculations, the models by HTG04 show a strong trend toward self-regulation that has also been found in the 3D chemodynamical $N$-body-SPH models of Berczik et al. (2002) including dark matter. The PWI04 models exhibit no strong starbursts as the moderate variation in their star formation rates shows (Figs. 4 and 6 in PWI04). In contrast to them we were looking for strong variations that are rarer, but that exist (van Zee 2001).

Another interesting example of SFR variability is the SPH simulation of Struck (2005, hereafter S05), who investigated the evolution of a disk galaxy of about $2 \times 10^{11} M_{\odot}$. The gaseous component in this simulation was subject to star formation, stellar feedback (by SNII) and dissipation (radiative cooling). S05 finds a strongly variable star formation with two modes: local, off-centre gas concentrations can lead to local, "premature" bursts preventing the system from a global starburst. The result is an incoherent star formation pattern with an irregular, mildly variable SFR. Sometimes, however, large-scale motions coherently funnel matter into the central region where a strong starburst is then ignited (cf. Figs. 1 and A1 in S05). The related timescale is about twice the free-fall timescale of a particle at $2-3 \mathrm{kpc}$ above the plane. This burst mode corresponds physically to the oscillatory "breathing" mode seen in our calculations, though the masses of the models differ by one order of 
magnitude. Similarly, the burst duration (about $10 \mathrm{Myr}$ ) is comparable to the burst duration in our models. On the other hand, our simple model is unable to resolve purely local effects leading to incoherent star formation modes.

Though a detailed comparison between our simple model and the different complex numerical simulations is unreasonable because of differences in implemented physics and in initial conditions, the qualitative agreement is obvious: the bursts essentially stem from the oscillations of the global dynamics. The gas simply has to follow them, so the starbursts could appear in any system. The tight coupling between the large-scale dynamics and the occurrence of global (strong) starbursts is corroborated in all the different studies, by this motivating and strengthening our ansatz.

\subsection{A speculation about bursts in interacting galaxies...}

By definition our model refers to isolated systems. However, the initial conditions leading to a collapse of the system could also stem from galaxy interactions. Therefore, one might speculate about the possible implications for interacting galaxies.

An important result of our simulations is the strong coupling of the SFR to the dynamics (with the discussed exception of linear Schmidt law without feedback term). To create a starburst, the gas density must be strongly enhanced, otherwise the self-regulation prevents large SFR variations. The availability of large amounts of gas alone is not sufficient for a starburst as the example of poststarburst E+A galaxies with a large amount of HI gas demonstrated (Buyle et al. 2006).

For interacting galaxies, one might speculate that only those perturbations leading to structural rearrangements, e.g. a substantial inflow of gas or a merger, end up in a starburst. Whether galaxies produce sufficient density enhancements, hence a starburst, depends not only on the existence of a proper perturbation (like an interaction or an internal bar) but also on the internal dynamical stability of the involved galaxies. This might explain the wide variety of star formation responses found in simulations of interacting galaxies (e.g. in Di Matteo et al. 2007).

\subsection{Simplifications of the model}

Describing galaxy evolution by a few equations always requires simplifications. Here we want to comment on a few simplifications (or neglections).

Dynamics. The dynamics of the system is summarily described by a mean radius and its temporal change. A decomposition into several structural components (like a disk-bulge-halo structure) is beyond the limits of this model. Moreover, density gradients or even an asphericity are not considered. An extension of our simple model might be possible. However, with respect to structure formation, we think that the related physics are too complex to be condensed reliably into another "simple" equation.

Implicitly we also assumed that the dynamics of the different baryonic components can be modelled by the same mean radius. This need not to be true, of course. Strictly speaking this is only valid when the dynamics of stars and gas is either subject to the same acceleration terms (e.g. similar pressure/velocity dispersion terms) and/or when the self-gravity of the stars is not important for the dynamical evolution of the gas. The latter is the case in gas-rich galaxies or in dark-matter-dominated potentials (when the self-gravity term remains unimportant). Since we are interested here in starburst galaxies that are mainly gas-rich dwarf galaxies, the restriction to gas-dominated stages seems reasonable. The dynamical equations can be easily extended to several distinct components, if required.

ISM description. In our approach we adopted a single ISM phase. An extension to a multi-phase ISM model like in Köppen et al. (1998) would be possible and is envisaged. However, we do not expect significant changes, because the timescales related to the additional multi-phase ISM processes (like condensation, evaporation) are separated well from the other timescales: they are much longer than the dominant heating and cooling processes, so that thermal equilibrium is established rapidly and independently of the fractional ratio of clouds and intercloud gas. On the other hand, evaporation and condensation of clouds occur much faster than the gas consumption due to star formation. Thus, we expect that the system would again break down in a hierarchy of well-separated equilibria, and the related equilibrium SFR would thus still be governed by the equilibrium temperature in the cloud phase.

A new effect that arises for a clumpy ISM is another energy dissipation process. In the case of a diffuse ISM, dissipation is mainly driven by radiative cooling; however, in case of a system composed of molecular clouds, inelastic cloud collisions provide a "cooling" mechanism dissipating the kinetic energy related to the motion of the clouds. Though the scaling of this dissipation process is identical to radiative cooling (i.e. $\propto g^{2}$ ), the related timescales differ strongly. In general, cloud-cloud collisions operate on a much longer timescale than radiative cooling or even the dynamics. Thus, the dynamical evolution of a cloud system might operate not only on a dynamical timescale, but also on a dissipational timescale which then directly affects the SFR. We will discuss the implications in a forthcoming paper in detail.

Heating. For the heating we only considered massive stars and instantaneous feedback. Though massive stars are considered to be a major source of ISM heating, e.g. with respect to turbulence, other sources might be interesting, too. For example, type I supernovae provide a nonlocal and a non-instantaneous energy source. Especially, the delayed energy feedback might be an important energy source after star formation has ceased. Similarly, the energy injected by an AGN might be an important energy source, too.

\section{Summary and conclusions}

We investigated the evolution of star-forming dwarf galaxies by means of an extended one-zone model. In contrast to previous one-zone models, the dynamics of the galaxy has been taken into account by an additional equation of motion for the mean radius of the galaxy. Comparison with SPH models shows that this rather approximate treatment is reasonable and provides a sensible description. This approach allows for a coupling between the dynamical state of the galaxy and its internal properties, such as star formation activity and the thermal state of the interstellar gas. Here we focussed especially on the conditions under which starburst episodes could occur in isolated galaxies.

We find that the seemingly complicated system of equations for the numerous physical variables (radius, radial speed, gas mass, star mass, thermal energy) breaks down into quite a clearly separated system for the internal conditions of the galaxy and the dynamical aspects that evolve more slowly. Thus, star formation and other internal processes are strongly governed by the 
dynamical state. In particular, the total SFR follows the evolution of the volume directly, if it depends nonlinearly on the local gas density. On the other hand, the dynamics are only very weakly influenced by the internal state, because the gas temperature remains well below virial temperature, primarily because of the strong rise of the radiative cooling function near $10^{4} \mathrm{~K}$. The consequence is that the evolution of the dynamical state of the galaxy remains fairly robust against changes and details in the internal condition, specifically the details of the prescriptions for star formation. We also expect that the inclusion of a multiphase description of the ISM would not upset this characteristic.

The evolution of the total SFR is thus dominated by the damped virial oscillations that cause enhancements of the SFR whenever the galaxy is in a more compressed state. This main type of quasi-periodic starbursts is related to the dynamical timescale of the system. Typically, the star formation variations follow the variations in the gas density induced by decaying virial oscillations. Because of the short heating and cooling timescales the established SFR remains close to the equilibrium SFR determined by the current mean gas density. Modifications in the adopted star formation modes, i.e. assuming both spontaneous and induced star formation/positive feedback, give qualitatively similar results. Induced star formation neither leads to a fundamentally different response nor causes a global conflagration. Variations in the IMF or introduction of a time-variable IMF similarly do not change that picture. In all cases, the variations in the SFR can be appreciable, amounting to peak values up to 10 times the average value.

A second type of burst occurs for low gas densities and temperatures beyond $10^{4} \mathrm{~K}$. This mode is not related to the dynamical evolution, but an instability operating when the cooling function drops with increasing temperature. The latter works for some temperature regimes (related to $\mathrm{H}$ and $\mathrm{He}$ line emission) above $10^{4} \mathrm{~K}$, which can only be reached when there is a very low gas density.

Since the first type of bursts is governed by the evolution of the dynamical state of the galaxy, it also seems plausible to discuss the results of our simplified models for isolated galaxies in other contexts, too. Virial oscillations following structural changes induced by galaxy interactions would also lead to an enhancement of the SFR, thus providing an additional mechanism that shapes starbursts. Alternatively, if an interaction does not result in a substantial mass redistribution, self-regulation might prevent a starburst.

Acknowledgements. We thank Jay Gallagher, Simone Recchi, and Shu-ichiro Inutsuka for stimulating discussions on starbursts and induced star formation. We are very grateful to the referee, Curt Struck, for detailed and fruitful comments that greatly improved the paper. C.T. is grateful to the Observatoire de Strasbourg for hospitality and support within the visitor programme.

\section{References}

Bekki, K. 2008, MNRAS, 338, L10

Berczik, P., Hensler, G., Theis, Ch., \& Spurzem, R. 2002, Ap\&SS, 281, 297 Binney, J., \& Tremaine, S. 1987, Galactic Dynamics (Princeton Univ. Press) Blitz, L., \& Rosolowsky, E. 2004, ApJ, 612, L29

Böhringer, H., \& Hensler, G. 1989, A\&A, 215, 147 Burkert, A. 1995, ApJ, 447, L25

Buyle, P., Michielsen, D., De Rijcke, S., et al. 2006, ApJ, 649, 163 Dalgarno, A., \& McCray, R. A. 1972, ARA\&A, 10, 375

Di Matteo, P., Combes, F., Melchior, A.-L., \& Semelin, B. 2007, A\&A, 468, 61 Dohm-Palmer, R. C., Skillman, E., Mateo, M., et al. 2002, AJ, 123, 813

Ehlerová, S., Palouš, J., Theis, Ch., \& Hensler, G. 1997, A\&A, 328, 121 Gallagher, J. S., \& Hunter, D. 1984, ARA\&A, 22, 37

Gerola, H., \& Seiden, P. E. 1978, ApJ, 223, 129

Greggio, L., Marconi, G., Tosi, M., \& Focardi, P. 1993, AJ, 105, 894

Harfst, S., Theis, Ch., \& Hensler, G. 2006, A\&A, 449, 509

Hensler, G., Theis, Ch., \& Gallagher, J. 2004, A\&A, 426, 25

Hosokawa, T., \& Inutsuka, S. 2005, ApJ, 623, 917

Hosokawa, T., \& Inutsuka, S. 2006, ApJ, 646, 240

Ikeuchi, S., \& Tomita, H. 1983, PASJ, 35, 77

Köppen, J., Theis, Ch., \& Hensler, G. 1995, A\&A, 296, 99, KTH95

Köppen, J., Theis, Ch., \& Hensler, G. 1998, A\&A, 331, 524

Köppen, J., Weidner, C., \& Kroupa, P. 2007, MNRAS, 375, 673

Nikolic, B., Cullen, H., \& Alexander, P. 2004, MNRAS, 355, 874

Pelupessy, F. I., van der Werf, P. P., \& Icke, V. 2004, A\&A, 422, 55

Press, W. H., Teukolsky, S. A., Vetterling, W. T., \& Flannery, B. P. 1992,

Numerical Recipes (Cambridge: Cambridge University Press)

Quillen, A. C., \& Bland-Hawthorn, J. 2008, MNRAS, 386, 2227

Samland, M., Hensler, G., \& Theis, Ch. 1997, ApJ, 476, 544

Sanders, D. B., \& Mirabel, I. F. 1996, ARA\&A, 34, 749

Scalo, J., \& Struck-Marcell, C. 1986, ApJ, 301, 77

Struck, C. 2005, in Proc. of Starbursts: From 30 Doradus to Lyman Break Galaxies, Cambridge, UK, ed. R. de Grijs, \& R. M. Gonzalez-Delgado, Ap\&SS, 329, 163

Struck, C. 2006, in Astrophysics Update 2, ed. J. Mason, 115

Theis, Ch., \& Hensler, G. 1993, A\&A, 280, 85

Theis, Ch., Burkert, A., \& Hensler, G. 1992, A\&A, 265, 465

Weidner, C., \& Kroupa, P. 2005, ApJ, 625, 754

van Zee, L. 2001, AJ, 121, 2003 